\title{
Sufficient conditions for regularity and uniqueness of a 3D nematic liquid crystal model.
}

\author{
F. Guillén-González ${ }^{1}$, M. A. Rodríguez-Bellido ${ }^{1}$, M. A. Rojas-Medar ${ }^{2}$
}

5th July 2005

${ }^{1}$ Dpto. de Ecuaciones Diferenciales y Análisis Numérico, Universidad de Sevilla, Aptdo. 1160, 41080 Sevilla, Spain. e-mails: guillen@us.es, angeles@us.es

${ }^{2}$ Dpto. de Matemática Aplicada, IMECC-UNICAMP, C.P. 6065, 13083-970, CampinasSP, Brazil. e-mail: marko@ime.unicamp.br

\begin{abstract}
In [3], L. Berselli showed that the additional regularity hypothesis for the velocity gradient $\nabla \mathbf{u} \in L^{\frac{2 q}{2 q-3}}\left(0, T ; \mathbf{L}^{q}(\Omega)\right)$, for some $q \in(3 / 2,+\infty]$, implies the strong regularity for the weak solutions of the Navier-Stokes equations. In this work, we prove that such hypothesis is also sufficient in order to obtain the strong solution for a nematic Liquid Crystal model (a coupled system of velocity $\mathbf{u}$ and orientation crystals vector $\mathbf{d}$ ) when periodic boundary conditions for $\mathbf{d}$ are considered. For Neumann and Dirichlet boundary conditions, we obtain the same result only for the cases of $q \in[2,3]$, whereas in the cases $q \in(3 / 2,2) \cup(3,+\infty]$, we also need to impose an additional regularity hypothesis for $\mathbf{d}$ (either on $\nabla \mathbf{d}$ or $\Delta \mathbf{d}$ ).

On the other hand, when the following hypothesis for $\mathbf{u}$ of Serrin's type is imposed $([18]): \mathbf{u} \in L^{\frac{2 p}{p-3}}\left(0, T ; \mathbf{L}^{p}(\Omega)\right)$ for some $p \in(3,+\infty]$, we can obtain strong regularity only in the case of periodic boundary conditions for $\mathbf{d}$. For Neumann or Dirichlet boundary conditions, additional regularity for $\mathbf{d}$ must be imposed in all cases.
\end{abstract}


Palabras clave: Liquid Crystal system, sufficient hypothesis of regularity, strong solution, uniqueness, regularity criterion.

AMS subject classification: 35Q30.

\section{Introduction and main results}

\subsection{The model}

In this work, we are interested in the study of regularity for a Navier-Stokes type model for incompressible fluids that takes into account the crystallinity of the fluid molecules. Thus, the Navier-Stokes equations are coupled with the Ginzburg-Landau equations, obtaining a nematic Liquid Crystal model.

The unknowns are the solenoidal velocity $\mathbf{u}(t, \mathbf{x})$, the pressure of the fluid $p(t, \mathbf{x})$, and the director field $\mathbf{d}(t, \mathbf{x})$, that represents the orientation of the liquid crystal molecules. Moreover, we suppose that the fluid is confined in a three-dimensional smooth enough domain $\Omega$.

Here, we present an Ericksen-Leslie type formulation, introduced by F. H. Lin in [11] and analyzed by F. H. Lin \& C. Liu in [12]. In fact, the model is a penalized one where the restriction $|\mathbf{d}| \leq 1$ appears as a consequence of a maximum principle for the Ginzburg-Landau equations, which is approximated by:

$$
\mathbf{f}_{\delta}(\mathbf{d})=\frac{1}{\delta^{2}}\left(|\mathbf{d}|^{2}-1\right) \mathbf{d}
$$

where $|\mathbf{d}|$ denotes the euclidean norm in $\mathbb{R}^{3}$ and $\delta>0$ is a penalization parameter. This penalization function has a potential structure, i. e. there exists a potential function $F_{\delta}(\mathbf{d})=\frac{1}{\delta^{2}}\left(|\mathbf{d}|^{2}-1\right)^{2}$ such that $\mathbf{f}_{\delta}(\mathbf{d})=\nabla_{\mathbf{d}}\left(F_{\delta}(\mathbf{d})\right)$ for all $\mathbf{d} \in \mathbb{R}^{3}$. Denoting $Q=$ $(0, T) \times \Omega$ and $\Sigma=(0, T) \times \partial \Omega$, where $\Omega$ a smooth enough domain and $\partial \Omega$ its boundary, 
the model can be written as:

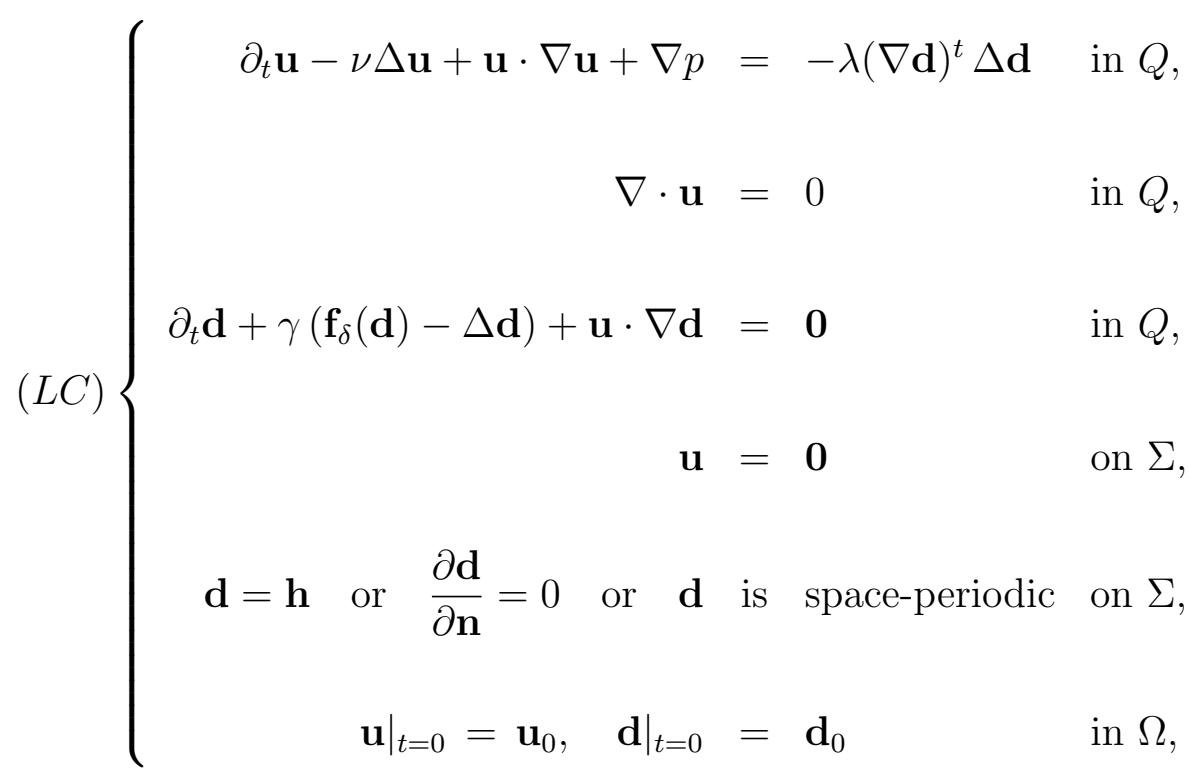

As for the coefficients, $\nu>0$ is the fluid viscosity, $\lambda>0$ is the elasticity constant and $\gamma>0$ is a relaxation in time constant.

Now we explain the boundary conditions: for the velocity $\mathbf{u}$ we always consider the non-slip boundary condition $(\mathbf{u}=\mathbf{0}$ on $\Sigma)$; for the orientation vector $\mathbf{d}$, the condition used for the model derivation as a penalized model [12] is of non-homogeneous Dirichlet type:

$$
\mathbf{d}=\mathbf{h} \quad \text { on } \Sigma \quad \text { such that }|\mathbf{h}| \leq 1 \text { in } \Sigma \text {. }
$$

There exists some others works where the boundary condition considered is of homogeneous Neumann type [15] :

$$
\partial_{\mathbf{n}} \mathbf{d}=\mathbf{0} \quad \text { on } \Sigma \text {. }
$$

jointly with the following free-slip boundary conditions for $\mathbf{u}$ :

$$
\mathbf{u} \cdot \mathbf{n}=0, \quad(\nabla \times \mathbf{u}) \times \mathbf{n}=\mathbf{0} \quad \text { on } \Sigma .
$$

Finally, we will also consider periodic boundary conditions for $\mathbf{d}$. In this case, the domain $\Omega$ is a square $(n=2)$ or a cube $(n=3)$.

\subsection{Known results}

For the Navier-Stokes equations, several works have studied the influence of the additional hypothesis over the velocity $\mathbf{u}$ in order to turn a weak solution $\mathbf{u}$ into a strong solu- 
tion, or to obtain uniqueness. Serrin proved in 1962 that if $\mathbf{u}$ is a Leray-Hopf weak solution belonging to $L^{\alpha}\left(0, T ; \mathbf{L}^{\gamma}\left(\mathbb{R}^{3}\right)\right)$ where $2 / \alpha+3 / \gamma \leq 1$ for $2<\alpha<+\infty$ and $3<\gamma<+\infty$, then the solution $\mathbf{u} \in C^{\infty}\left((0, T) \times \mathbb{R}^{3}\right)$. The same tesis was improved by other authors imposing weaker hypothesis, for instance Giga supposing $\mathbf{u} \in C\left([0, T] ; \mathbf{L}^{3}\left(\mathbb{R}^{3}\right)\right)$, [7]. There are also works were the weak regularity of the velocity is improved imposing regularity criteria over the gradient of velocity (the case of the whole space was proved in [1] and the bounded domain case in [3]) or the pressure (see for example, [2, 4]). Or even, only supposing that one component of the velocity field belongs to $L^{\infty}\left((0, T) \times \mathbb{R}^{3}\right.$ ) (see [10]). Recently, in [17], several different criteria on $\mathbf{u}$ or over some components of $\mathbf{u}$ are considered with the same objective.

In our case, a coupled system of $\mathbf{u}$ and $\mathbf{d}$ is treated in a bounded domain. Here, we analyze the cases where only additional hypothesis on $\mathbf{u}$ are necessary to provide the strong regularity for $(\mathbf{u}, \mathbf{d})$. Sometimes, this will be possible (see results below when imposing (H2) hypothesis for $\nabla \mathbf{u}$ with $q \in[2,3])$. Otherwise, an additional regularity on d (the crystal orientation field) will be imposed.

We denote by $\mathbf{H}$ and $\mathbf{V}$ the space of type $L^{2}$ and $H^{1}$ respectively, associated to the incompressibility and adherence velocity conditions:

$$
\begin{aligned}
& \mathbf{H}=\left\{\mathbf{u} \in \mathbf{L}^{2}(\Omega): \nabla \cdot \mathbf{u}=0, \mathbf{u} \cdot \mathbf{n}_{\mid \partial \Omega}=0\right\} \\
& \mathbf{V}=\left\{\mathbf{u} \in \mathbf{H}^{1}(\Omega): \nabla \cdot \mathbf{u}=0, \mathbf{u}_{\mid \partial \Omega}=0\right\}
\end{aligned}
$$

Observe that the boldface is used to denote the vectorial spaces in order to distinguish from the scalar spaces.

On the Liquid Crystal model (LC), under compatibility hypothesis:

$$
\left|\mathbf{d}_{0}\right| \leq 1, \quad|\mathbf{h}| \leq 1 \quad \text { a. e. in } \Sigma
$$

and (weak) regularity hypothesis for the data:

$$
\mathbf{u}_{0} \in \mathbf{H}, \quad \mathbf{d}_{0} \in \mathbf{H}^{1}(\Omega), \quad \mathbf{h} \in L^{\infty}\left(0, T ; \mathbf{H}^{1 / 2}(\partial \Omega)\right) \cap L^{2}\left(0, T ; \mathbf{H}^{3 / 2}(\partial \Omega)\right),
$$

it is known $([12])$ the existence of weak solution global in time, that is a solution with the 
following regularity:

$$
\left\{\begin{array}{l}
\mathbf{u} \in L^{\infty}(0, T ; \mathbf{H}) \cap L^{2}(0, T ; \mathbf{V}) \\
\mathbf{d} \in L^{\infty}\left(0, T ; \mathbf{H}^{1}(\Omega)\right) \cap L^{2}\left(0, T ; \mathbf{H}^{2}(\Omega)\right), \quad|\mathbf{d}| \leq 1
\end{array}\right.
$$

In a previous work, some of the authors of this work obtained in [9] the existence of a weak solution global in time (without restrictions for the data) for the limit problem (when $\delta \rightarrow 0$ in $(L C)$ ).

Note that if $\Omega \subseteq \mathbb{R}^{2}$, we know the existence and uniqueness of smooth solution global in time, see [12]. For this, we focus our study in $\Omega \subseteq \mathbb{R}^{3}$.

On the other hand, under the hypothesis of strong regularity for the data:

$$
\mathbf{u}_{0} \in V, \quad \mathbf{d}_{0} \in \mathbf{H}^{2}(\Omega), \quad \mathbf{h} \in L^{\infty}\left(0, T ; \mathbf{H}^{3 / 2}(\partial \Omega)\right) \cap L^{2}\left(0, T ; \mathbf{H}^{5 / 2}(\partial \Omega)\right),
$$

it is known the existence and uniqueness of strong solution with restrictions (global in time for small data or local in time for any data), that is, the solution has the following regularity:

$$
\left\{\begin{array}{l}
\mathbf{u} \in L^{\infty}\left(0, T_{*} ; \mathbf{V}\right) \cap L^{2}\left(0, T_{*} ; \mathbf{H}^{2}(\Omega)\right) \\
\mathbf{d} \in L^{\infty}\left(0, T_{*} ; \mathbf{H}^{2}(\Omega)\right) \cap L^{2}\left(0, T_{*} ; \mathbf{H}^{3}(\Omega)\right),
\end{array}\right.
$$

with $T_{*} \leq T$ (small enough) or $T_{*}=T$ for small data.

Finally, from the previous regularity and under the hypothesis:

$$
\partial_{t} \mathbf{h} \in L^{\infty}\left(0, T ; \mathbf{H}^{-1 / 2}(\partial \Omega)\right) \cap L^{2}\left(0, T ; \mathbf{H}^{1 / 2}(\partial \Omega)\right),
$$

it is easy to obtain (a posteriori) the following additional regularity (see Lemma 2.3):

$$
\left\{\begin{array}{l}
\partial_{t} \mathbf{u} \in L^{2}(0, T ; \mathbf{H}), \quad p \in L^{2}\left(0, T ; H^{1}(\Omega)\right) \\
\partial_{t} \mathbf{d} \in L^{\infty}\left(0, T ; \mathbf{L}^{2}(\Omega)\right) \cap L^{2}\left(0, T ; \mathbf{H}^{1}(\Omega)\right) .
\end{array}\right.
$$




\subsection{Main new results}

Our aim is to elucidate sufficient regularity hypothesis for $\mathbf{u}$ in order to obtain strong regularity global in time (and uniqueness) for the solution of $(L C)$, without imposing additional regularity hypothesis for $\mathbf{d}$ (if it is possible). In this way, we impose two kinds of conditions for $\mathbf{u}$ :

$(H 1) \quad \nabla \mathbf{u} \in L^{\frac{2 q}{2 q-3}}\left(0, T ; \mathbf{L}^{q}(\Omega)\right) \quad$ for some $q: 3 / 2<q \leq \infty$

or

$(H 2) \quad \mathbf{u} \in L^{\frac{2 p}{p-3}}\left(0, T ; \mathbf{L}^{p}(\Omega)\right) \quad$ for some $3<p \leq+\infty$.

When necessary, we will impose the following two kinds of additional regularity for $\mathbf{d}$ :

(H3) $\quad \nabla \mathbf{d} \in L^{\frac{2 p}{p-3}}\left(0, T ; \mathbf{L}^{p}(\Omega)\right) \quad$ for some $p: 3 \leq p \leq+\infty$,

or

(H4) $\quad \Delta \mathbf{d} \in L^{\frac{2 q}{2 q-3}}\left(0, T ; \mathbf{L}^{q}(\Omega)\right)$ for some $q: 3 / 2<q \leq+\infty$.

Observe that hypothesis (H2) is the Serrin's condition (see [18]) and hypothesis $(H 1)$ was introduced by Berselli in [3] when $q \geq 3$ (the case $q \in(3 / 2,3)$ is implied by the Serrin's condition). Both hypothesis guarantee the strong regularity and uniqueness for the Navier-Stokes equations.

Next, we state the main results, indicating the sections where each result will be proved. Always, the hypothesis (3), (5) and (6) are imposed on the data.

Theorem 1.1 (Non-homogeneous Dirichlet or homogeneous Neumann cases) Let $(\mathbf{u}, \mathbf{d})$ be a weak solution of $(L C)$ in $(0, T)$ such that $\nabla \mathbf{u}$ verifies $(H 1)$ with exponent $q: 2 \leq q \leq 3$. Then, $(\mathbf{u}, \mathbf{d})$ is the unique strong solution of $(L C)$ in $(0, T)$.

The proof will be made using Prodi's type estimates in a decoupled manner, first for $\mathbf{d}$, and afterwards using this regularity, for $\mathbf{u}$ (see Section 2).

Theorem 1.2 (Other cases) If $(\mathbf{u}, \mathbf{d})$ is a weak solution of $(L C)$ such that $\mathbf{u}$ verifies either (H1) or (H2), and $\mathbf{d}$ verifies either (H3) or (H4), then $(\mathbf{u}, \mathbf{d})$ is the unique strong solution of $(L C)$. 
In this case (see Section 3), we use Ladyzhenskaya's type estimates for $\mathbf{u}$ and $\mathbf{d}$ in a coupled manner.

Theorem 1.3 (Periodic boundary conditions for $\mathbf{d})$ Let $(\mathbf{u}, \mathbf{d})$ be a weak solution of (LC) with periodic boundary conditions for $\mathbf{d}$ such that $\mathbf{u}$ verifies either (H2) for $p>3$ or $(H 1)$. Then, $(\mathbf{u}, \mathbf{d})$ is the unique strong solution of $(L C)$.

The proof (see Section 4) is based on the obtention of the following non-hilbertian regularity for $\mathbf{d}, \nabla \mathbf{d} \in L^{3}\left(0, T ; \mathbf{L}^{9}(\Omega)\right)$, and the application of Theorem 1.2.

Theorem 1.4 (Uniqueness) Suppose that the data verify the weak regularity given by (4) and the constraints (3), and $(\mathbf{u}, \mathbf{d})$ is a weak solution of $(L C)$ such that $\mathbf{u}$ verifies either (H2) or (H1), and $\mathbf{d}$ verifies either (H3) or (H4). Then, this solution coincides with any weak solution (associated to the same data).

Proof: See Section 5 when $(H 1)$ condition is imposed, and Section 6 for $(H 2)$ condition.

Remark 1.1 Observe that in Theorems 1.1, 1.2 and 1.3, strong regularity is obtained assuming strong regularity on the data $\left(\mathbf{u}_{0}, \mathbf{d}_{0}, \mathbf{h}\right)$. But the uniqueness result (Theorem 1.4) is obtained only assuming weak regularity on the data (and additional regularity on weak solutions). However, Theorems 1.1 and 1.3 give uniqueness results (a posteriori) under less additional regularity hypothesis over the weak solution than in Theorem 1.4 (only with additional regularity for $\mathbf{u}$ and not for $\mathbf{d}$ ).

Remark 1.2 (Comparison between the hypothesis $(H 1)$ and $(H 2)$ for $\mathbf{u}$, and (H3) and (H4) for d)

Thanks to the Sobolev's embeddings, the hypothesis (H1) (resp. (H4)) for $3 / 2<q<3$ implies the hypothesis (H2) (resp. (H3)) for $3<p<+\infty$. In particular, the hypothesis (H2) guarantees the strong regularity for the solution of the Navier-Stokes equations, see for instance [18]. 


\section{Proof of Theorem 1.1}

We want to use the additional regularity hypothesis $(H 1)$ for $\nabla \mathbf{u}$ (with $2 \leq q \leq 3$ ), in order to obtain strong regularity for $(L C)$ without additional regularity hypothesis for $\mathbf{d}$.

Due to the particular structure of the equations for $\mathbf{u}$, specially the strongly nonlinear term $(\nabla \mathbf{d})^{t} \Delta \mathbf{d}$ in the $\mathbf{u}$-system, now it will be necessary to obtain greater regularity for d.

An essential characteristic of the problem for $\mathbf{d}$ (given $\mathbf{u}$ ) is the following weak maximum principle:

Lemma 2.1 (Maximum principle) Given $\mathbf{u} \in L^{2}(0, T ; \mathbf{V}) \cap L^{\infty}(0, T ; \mathbf{H})$ and $\mathbf{d}_{0} \in$ $\mathbf{L}^{2}(\Omega)$ with $\left|\mathbf{d}_{0}\right| \leq 1$ a. e. in $\Omega$, let $\mathbf{d}$ be any weak solution for the $\mathbf{d}$-problem (either in the non-homogeneous Dirichlet case, $\left.\mathbf{d}\right|_{\Sigma}=\mathbf{h}$ with $|\mathbf{h}| \leq 1$ a. e. on $\Sigma$, or the homogeneous Neumann case, $\left.\partial_{\mathbf{n}} \mathbf{d}\right|_{\Sigma}=\mathbf{0}$ or the space-periodic case), i.e. $\mathbf{d} \in L^{2}\left(0, T ; \mathbf{H}^{1}(\Omega)\right) \cap$ $L^{\infty}\left(0, T ; \mathbf{L}^{2}(\Omega)\right)$ such that for all $\mathbf{e} \in \mathbf{H}_{0}^{1}(\Omega) \quad$ or $\left.\mathbf{H}^{1}(\Omega)\right)$ :

$$
\begin{aligned}
& \left\langle\partial_{t} \mathbf{d}, \mathbf{e}\right\rangle+((\mathbf{u} \cdot \nabla) \mathbf{d}, \mathbf{e})+\left(\mathbf{f}_{\delta}(\mathbf{d}), \mathbf{e}\right)+(\nabla \mathbf{d}, \nabla \mathbf{e})=0, \\
& \mathbf{d}(0)=\mathbf{d}_{0}
\end{aligned}
$$

Then, $|\mathbf{d}(\mathbf{x}, t)| \leq 1$ a.e. in $Q$.

Proof: See [5]. For reader's convenience completeness, a proof is included in Appendix A.1.

In particular, $\left|\mathbf{f}_{\delta}(\mathbf{d})\right| \leq C$ and $\left|\nabla \mathbf{f}_{\delta}(\mathbf{d})\right| \leq C|\nabla \mathbf{d}|$, where $C$ is a constant (depending on $\left.1 / \delta^{2}\right)$.

In the proof of Theorem 1.1, we distinguish between two kinds of boundary conditions: homogeneous Neumann boundary conditions, that is, $\left.\left(\partial_{\mathbf{n}} \mathbf{d}\right)\right|_{\partial \Omega}=\mathbf{0}$, and nonhomogeneous Dirichlet boundary conditions, that is, $\left.\mathbf{d}\right|_{\partial \Omega}=\mathbf{h}$ (the space-periodic case will be easier). 


\subsection{Homogeneous Neumann boundary conditions}

Notice that for homogeneous Neumann boundary conditions, we are going to consider the following equivalent norms:

$$
\begin{aligned}
\|\mathbf{d}\|_{H^{1}(\Omega)} & \sim\left(\|\nabla \mathbf{d}\|_{L^{2}(\Omega)}^{2}+\|\mathbf{d}\|_{L^{2}(\Omega)}^{2}\right)^{1 / 2} \\
\|\mathbf{d}\|_{H^{2}(\Omega)} & \sim\left(\|\Delta \mathbf{d}\|_{L^{2}(\Omega)}^{2}+\|\mathbf{d}\|_{L^{2}(\Omega)}^{2}\right)^{1 / 2} \\
\|\mathbf{d}\|_{H^{3}(\Omega)} & \sim\left(\|\nabla(\Delta \mathbf{d})\|_{L^{2}(\Omega)}^{2}+\|\mathbf{d}\|_{H^{2}(\Omega)}^{2}\right)^{1 / 2}
\end{aligned}
$$

The proof of existence of weak solution ([12]) is based on the restriction $|\mathbf{d}| \leq 1$ in $Q$ (consequence of a weak maximum principle, see Theorem 2.1 for the equation verified by $|\mathbf{d}|^{2}$ ), and the following inequalities:

$$
\begin{aligned}
& \frac{1}{2} \frac{d}{d t}\|\mathbf{d}\|_{L^{2}}^{2}+\gamma\|\nabla \mathbf{d}\|_{L^{2}}^{2} \leq C \\
\frac{1}{2} \frac{d}{d t}\left(\|\mathbf{u}\|_{L^{2}}^{2}\right. & \left.+\lambda \gamma\|\nabla \mathbf{d}\|_{L^{2}}^{2}+\lambda \gamma F_{\delta}(\mathbf{d})\right) \\
& +\nu\|\nabla \mathbf{u}\|_{L^{2}}^{2}+\lambda \gamma\left\|\left(\mathbf{f}_{\delta}(\mathbf{d})-\Delta \mathbf{d}\right)\right\|_{L^{2}}^{2} \leq 0
\end{aligned}
$$

If we take $\Delta^{2} \mathbf{d}$ as a test function in the $\mathbf{d}$-system, integrating by parts in every term and a second time in the derivative in time term $\partial_{t} \mathbf{d}$, the boundary integrals cancel using that $\partial_{\mathbf{n}} \partial_{t} \mathbf{d}=0$ on $\left.\Sigma\right)$ :

$$
\begin{aligned}
& \frac{1}{2} \frac{d}{d t}\|\Delta \mathbf{d}\|_{L^{2}(\Omega)}^{2}+\gamma\|\nabla(\Delta \mathbf{d})\|_{L^{2}(\Omega)}^{2} \\
= & \gamma \int_{\Omega} \nabla \mathbf{f}_{\delta}(\mathbf{d}) \cdot \nabla(\Delta \mathbf{d}) d \Omega+\int_{\Omega} \nabla[(\mathbf{u} \cdot \nabla) \mathbf{d}] \nabla(\Delta \mathbf{d}) d \Omega \\
\leq & \gamma \int_{\Omega}\left|\nabla\left(\mathbf{f}_{\delta}(\mathbf{d})\right)\right||\nabla(\Delta \mathbf{d})| d \Omega+\int_{\Omega}|\nabla \mathbf{u}\|\nabla \mathbf{d}\| \nabla(\Delta \mathbf{d})| d \Omega \\
+ & \int_{\Omega}|\nabla \mathbf{u}|\left|D^{2} \mathbf{d}\right|^{2} d \Omega,
\end{aligned}
$$

where the last term on the right hand side is obtained as follows:

$$
\begin{aligned}
\left|\int_{\Omega}(\mathbf{u} \cdot \nabla) \nabla \mathbf{d} \cdot \nabla(\Delta \mathbf{d}) d \Omega\right| & =\left|-\int_{\Omega}(\nabla \mathbf{u} \cdot \nabla) \nabla \mathbf{d}: D^{2} \mathbf{d} d \Omega-\frac{1}{2} \int_{\Omega}(\mathbf{u} \cdot \nabla)\left(\left|D^{2} \mathbf{d}\right|^{2}\right) d \Omega\right| \\
& \leq \int_{\Omega}|\nabla \mathbf{u}|\left|D^{2} \mathbf{d}\right|^{2} d \Omega
\end{aligned}
$$


Applying the Young's inequality,

$$
\begin{aligned}
\frac{d}{d t}\|\Delta \mathbf{d}\|_{L^{2}(\Omega)}^{2} & +\gamma\|\nabla(\Delta \mathbf{d})\|_{L^{2}(\Omega)}^{2} \leq C\left(\int_{\Omega}\left|\nabla \mathbf{f}_{\delta}(\mathbf{d})\right|^{2} d \Omega\right. \\
& \left.+\int_{\Omega}|\nabla \mathbf{u}|^{2}|\nabla \mathbf{d}|^{2} d \Omega+\int_{\Omega}|\nabla \mathbf{u}|\left|D^{2} \mathbf{d}\right|^{2} d \Omega\right):=I_{1}+I_{2}+I_{3}
\end{aligned}
$$

If, moreover, we add (9) and $\gamma\|\mathbf{d}\|_{H^{2}(\Omega)}^{2}$ in both sides to the previous estimate, we obtain:

$$
\frac{d}{d t}\|\mathbf{d}\|_{H^{2}(\Omega)}^{2}+\gamma\|\mathbf{d}\|_{H^{3}(\Omega)}^{2} \leq I_{1}+I_{2}+I_{3}+\gamma\|\mathbf{d}\|_{H^{2}(\Omega)}^{2}+C
$$

From the definition of $\mathbf{f}_{\delta}(\mathbf{d})$, we have that:

$$
\left|\nabla\left(\mathbf{f}_{\delta}(\mathbf{d})\right)\right| \leq C\left(|\mathbf{d}|^{2}+1\right)|\nabla \mathbf{d}| \leq C|\nabla \mathbf{d}|
$$

hence

$$
I_{1} \leq \bar{\varepsilon}\|\nabla(\Delta \mathbf{d})\|_{L^{2}(\Omega)}^{2}+C_{\bar{\varepsilon}}\|\nabla \mathbf{d}\|_{L^{2}}^{2}
$$

The terms $I_{2}$ and $I_{3}$ can be controlled thanks to hypothesis $(H 1)$. Indeed, for any $q \geq 3 / 2$,

$$
\begin{aligned}
I_{3} & \leq C\|\nabla \mathbf{u}\|_{L^{q}(\Omega)}\left\|D^{2} \mathbf{d}\right\|_{L^{\frac{2 q}{q-1}}(\Omega)}^{2} \\
& \leq C\|\nabla \mathbf{u}\|_{L^{q}(\Omega)}\left\|D^{2} \mathbf{d}\right\|_{L^{2}(\Omega)}^{\frac{2 q-3}{q}}\left\|D^{2} \mathbf{d}\right\|_{H^{1}(\Omega)}^{\frac{3}{q}} \\
& \leq \bar{\varepsilon}\|\mathbf{d}\|_{H^{3}(\Omega)}^{2}+C_{\bar{\varepsilon}}\|\nabla \mathbf{u}\|_{L^{q}(\Omega)}^{\frac{2 q}{2 q-3}}\|\mathbf{d}\|_{H^{2}(\Omega)}^{2}
\end{aligned}
$$

We only have been able to get a good estimate of $I_{2}$ for $\mathbf{2} \leq \mathbf{q} \leq \mathbf{3}$. To this aim, we separate the cases $2<q<3, q=3$ and $q=2$.

- if $2<q<3$, we use the Sobolev's embedding $W^{1, \frac{6 q}{5 q-6}}(\Omega) \hookrightarrow L^{\frac{2 q}{q-2}}(\Omega)$, obtaining:

$$
\begin{aligned}
\int_{\Omega}|\nabla \mathbf{u}|^{2}|\nabla \mathbf{d}|^{2} d \Omega & \leq\|\nabla \mathbf{u}\|_{L^{q}(\Omega)}^{2}\|\nabla \mathbf{d}\|_{L^{\frac{2 q}{q-2}}(\Omega)}^{2} \\
& \leq C\|\nabla \mathbf{u}\|_{L^{q}(\Omega)}^{2}\|\nabla \mathbf{d}\|_{W^{1, \frac{6 q}{5 q-6}(\Omega)}}^{2} \\
& \leq C\|\nabla \mathbf{u}\|_{L^{q}(\Omega)}^{2}\|\nabla \mathbf{d}\|_{H^{1}(\Omega)}^{2 \frac{2 q-3}{q}}\|\nabla \mathbf{d}\|_{H^{2}(\Omega)}^{2\left(\frac{3}{q}-1\right)} \\
& \leq \bar{\varepsilon}\|\mathbf{d}\|_{H^{3}(\Omega)}^{2}+C_{\bar{\varepsilon}}\|\nabla \mathbf{u}\|_{L^{q}(\Omega)}^{\frac{2 q}{2 q-3}}\|\mathbf{d}\|_{H^{2}(\Omega)}^{2}
\end{aligned}
$$


- if $q=3$, then:

$$
\begin{aligned}
\int_{\Omega}|\nabla \mathbf{u}|^{2}|\nabla \mathbf{d}|^{2} d \Omega & \leq\|\nabla \mathbf{u}\|_{L^{3}(\Omega)}^{2}\|\nabla \mathbf{d}\|_{L^{6}(\Omega)}^{2} \\
& \leq C\|\nabla \mathbf{u}\|_{L^{3}(\Omega)}^{2}\|\nabla \mathbf{d}\|_{H^{1}(\Omega)}^{2}
\end{aligned}
$$

Observe that hypothesis $(H 1)$ in this case is $\nabla \mathbf{u} \in L^{2}\left(0, T ; \mathbf{L}^{3}(\Omega)\right)$.

- if $q=2$, we use the inequality (see Friedman [6]):

$$
\|\mathbf{f}\|_{L^{\infty}(\Omega)} \leq C\|\mathbf{f}\|_{H^{1}(\Omega)}^{1 / 2}\|\mathbf{f}\|_{H^{2}(\Omega)}^{1 / 2}
$$

obtaining:

$$
\begin{aligned}
\int_{\Omega}|\nabla \mathbf{u}|^{2}|\nabla \mathbf{d}|^{2} d \Omega & \leq\|\nabla \mathbf{u}\|_{L^{2}(\Omega)}^{2}\|\nabla \mathbf{d}\|_{L^{\infty}(\Omega)}^{2} \\
& \leq C\|\nabla \mathbf{u}\|_{L^{2}(\Omega)}^{2}\|\nabla \mathbf{d}\|_{H^{1}(\Omega)}\|\nabla \mathbf{d}\|_{H^{2}(\Omega)} \\
& \leq \bar{\varepsilon}\|\mathbf{d}\|_{H^{3}(\Omega)}^{2}+C_{\bar{\varepsilon}}\|\nabla \mathbf{u}\|_{L^{2}(\Omega)}^{4}\|\mathbf{d}\|_{H^{2}(\Omega)}^{2}
\end{aligned}
$$

Observe that hypothesis $(H 1)$ in this case is $\nabla \mathbf{u} \in L^{4}\left(0, T ; \mathbf{L}^{2}(\Omega)\right)$.

Then, for $2 \leq q \leq 3$ (and taking $\bar{\varepsilon}$ small enough), from (11), we obtain the following inequality:

$$
\frac{d}{d t}\|\mathbf{d}\|_{H^{2}(\Omega)}^{2}+\gamma\|\mathbf{d}\|_{H^{3}(\Omega)}^{2} \leq C\left(\|\nabla \mathbf{u}\|_{L^{p}(\Omega)}^{\frac{2 p}{2 p-3}}+1\right)\|\mathbf{d}\|_{H^{2}(\Omega)}^{2}+C
$$

that let us to conclude that $\mathbf{d} \in L^{\infty}\left(0, T ; \mathbf{H}^{2}(\Omega)\right) \cap L^{2}\left(0, T ; \mathbf{H}^{3}(\Omega)\right)$.

From that regularity, we can deduce the strong regularity for $(\mathbf{u}, p)$ and for $\partial_{t} \mathbf{d}$ taking into account the following Lemmas.

Lemma 2.2 Let $(\mathbf{u}, \mathbf{d})$ be a weak solution of $(L C)$ such that $\nabla \mathbf{u}$ has the additional regularity $(H 1)$, and $\mathbf{d} \in L^{\infty}\left(0, T ; \mathbf{H}^{2}(\Omega)\right) \cap L^{2}\left(0, T ; \mathbf{H}^{3}(\Omega)\right)$. Then $\mathbf{u} \in L^{\infty}\left(0, T ; \mathbf{H}^{1}(\Omega)\right) \cap$ $L^{2}\left(0, T ; \mathbf{H}^{2}(\Omega)\right)$.

Proof: See Appendix A.2. 
Lemma 2.3 Let $(\mathbf{u}, \mathbf{d})$ be a weak solution of $(L C)$ such that:

$$
\left\{\begin{array}{l}
\mathbf{d} \in L^{\infty}\left(0, T ; \mathbf{H}^{2}(\Omega)\right) \cap L^{2}\left(0, T ; \mathbf{H}^{3}(\Omega)\right) \\
\mathbf{u} \in L^{\infty}\left(0, T ; \mathbf{H}^{1}(\Omega)\right) \cap L^{2}\left(0, T ; \mathbf{H}^{2}(\Omega)\right) .
\end{array}\right.
$$

Then, $\partial_{t} \mathbf{d} \in L^{\infty}\left(0, T ; \mathbf{L}^{2}(\Omega)\right) \cap L^{2}\left(0, T ; \mathbf{H}^{1}(\Omega)\right)$ and $\partial_{t} \mathbf{u} \in L^{2}\left(0, T ; \mathbf{L}^{2}(\Omega)\right)$. Moreover, $p \in L^{2}\left(0, T ; H^{1}(\Omega)\right)$.

Proof: See Appendix A.3.

\subsection{Non-homogeneous Dirichlet boundary conditions}

We start considering a "good" lifting $\widetilde{\mathbf{d}}$ for the Dirichlet datum $\mathbf{h}$ in such a way that we could write a problem similar to $(L C)_{3}$ with homogeneous boundary data for $\mathbf{d}$ and that regular estimates of $H^{3}$-type for $\mathbf{d}$ can be made. We define this lifting function $\widetilde{\mathbf{d}}$ as the solution of the problem:

$$
\left\{\begin{array}{c}
\partial_{t} \widetilde{\mathbf{d}}+\gamma\left(\mathbf{f}_{\delta}(\mathbf{d})-\Delta \widetilde{\mathbf{d}}\right)=\mathbf{0} \quad \text { in } Q \\
\widetilde{\mathbf{d}}=\mathbf{h} \text { on } \Sigma,\left.\quad \widetilde{\mathbf{d}}\right|_{t=0}=\mathbf{d}_{0} \text { in } \Omega
\end{array}\right.
$$

The system (18) also verifies the maximum principle (since $\left|\mathbf{d}_{0}\right| \leq 1$ and $|\mathbf{h}| \leq 1$ ), hence $|\widetilde{\mathbf{d}}| \leq 1$ in $Q$. In particular, $\left|\mathbf{f}_{\delta}(\widetilde{\mathbf{d}})\right| \leq C$ and $\left|\nabla \mathbf{f}_{\delta}(\widetilde{\mathbf{d}})\right| \leq C|\nabla \widetilde{\mathbf{d}}|$.

For $\Omega$ smooth enough, thanks to the strong regularity of the data $\mathbf{h}$ and $\mathbf{d}_{0}$ given by (5) and (6), we have:

$$
\left\{\begin{array}{r}
\widetilde{\mathbf{d}} \in L^{\infty}\left(0, T ; \mathbf{H}^{2}(\Omega)\right) \cap L^{2}\left(0, T ; \mathbf{H}^{3}(\Omega)\right) \\
\partial_{t} \widetilde{\mathbf{d}} \in L^{\infty}\left(0, T ; \mathbf{L}^{2}(\Omega)\right) \cap L^{2}\left(0, T ; \mathbf{H}^{1}(\Omega)\right)
\end{array}\right.
$$

Then, the homogeneous problem verified by $\widehat{\mathbf{d}}=\mathbf{d}-\widetilde{\mathbf{d}}$ is written as follows:

$$
\left\{\begin{array}{r}
\partial_{t} \widehat{\mathbf{d}}+\gamma\left(\mathbf{f}_{\delta}(\mathbf{d})-\mathbf{f}_{\delta}(\widetilde{\mathbf{d}})-\Delta \widehat{\mathbf{d}}\right)+\mathbf{u} \cdot \nabla \mathbf{d}=\mathbf{0} \text { in } Q, \\
\left.\widehat{\mathbf{d}}\right|_{t=0}=\mathbf{0} \text { in } \Omega, \quad \widehat{\mathbf{d}}=\mathbf{0} \text { on } \Sigma .
\end{array}\right.
$$


Now, due to the homogeneous boundary condition verified by $\widehat{\mathbf{d}}$, we are going to consider the following equivalent norms:

$$
\begin{aligned}
\|\widehat{\mathbf{d}}\|_{H^{1}(\Omega)}^{2} & \approx\|\nabla \widehat{\mathbf{d}}\|_{L^{2}(\Omega)}^{2} \\
\|\widehat{\mathbf{d}}\|_{H^{2}(\Omega)}^{2} & \approx\|\Delta \widehat{\mathbf{d}}\|_{L^{2}(\Omega)}^{2} \\
\|\widehat{\mathbf{d}}\|_{H^{3}(\Omega)}^{2} & \approx\|\nabla(\Delta \widehat{\mathbf{d}})\|_{L^{2}(\Omega)}^{2}+\|\Delta \widehat{\mathbf{d}}\|_{L^{2}(\Omega)}^{2},
\end{aligned}
$$

If we take $\Delta^{2} \widehat{\mathbf{d}}$ as test a function in (19) and integrate by parts (twice in the $\partial_{t} \widehat{\mathbf{d}}$-term), we arrive at the expression:

$$
\begin{aligned}
\frac{1}{2} \frac{d}{d t} \| & \|\widehat{\mathbf{d}}\|_{L^{2}(\Omega)}^{2}+\gamma\|\nabla(\Delta \widehat{\mathbf{d}})\|_{L^{2}(\Omega)}^{2} \\
& =\gamma \int_{\Omega} \nabla\left[\mathbf{f}_{\delta}(\mathbf{d})-\mathbf{f}_{\delta}(\widetilde{\mathbf{d}})\right] \cdot \nabla(\Delta \widehat{\mathbf{d}}) d \Omega+\int_{\Omega} \nabla[(\mathbf{u} \cdot \nabla) \mathbf{d}] \cdot \nabla(\Delta \widehat{\mathbf{d}}) d \Omega \\
& :=J_{1}+J_{2}
\end{aligned}
$$

Notice that all the boundary integrals vanish. A formal justification is since $\left.\partial_{t} \widehat{\mathbf{d}}\right|_{\partial \Omega}$, $\left.\left(\mathbf{f}_{\delta}(\mathbf{d})-\mathbf{f}_{\delta}(\widetilde{\mathbf{d}})\right)\right|_{\partial \Omega}$ and $\left.(\mathbf{u} \cdot \nabla \mathbf{d})\right|_{\partial \Omega}=\mathbf{0}$, hence $\left.\Delta \widehat{\mathbf{d}}\right|_{\partial \Omega}=\mathbf{0}$ thanks to (19). A rigorous justification can be done with a Galerkin method, using the basis of laplacian eigenfunctions.

Thus,

$$
\begin{aligned}
J_{1} & \leq C \int_{\Omega}(|\nabla \widehat{\mathbf{d}}|+|\nabla \widetilde{\mathbf{d}}|)|\nabla \Delta \widehat{\mathbf{d}}| d \Omega \\
& \leq \bar{\varepsilon}\|\nabla(\Delta \widehat{\mathbf{d}})\|_{L^{2}(\Omega)}^{2}+C_{\bar{\varepsilon}}\left(\|\nabla \widehat{\mathbf{d}}\|_{L^{2}(\Omega)}^{2}+\|\nabla \widetilde{\mathbf{d}}\|_{L^{2}(\Omega)}^{2}\right)
\end{aligned}
$$

(here, we have used that $\left|\nabla \mathbf{f}_{\delta}(\mathbf{d})\right| \leq C|\nabla \mathbf{d}|$ and $\left.\left|\nabla \mathbf{f}_{\delta}(\widetilde{\mathbf{d}})\right| \leq C|\nabla \widetilde{\mathbf{d}}|\right)$. But, again integrating by parts in $J_{2}$ and taking into account that the boundary integrals cancel, we obtain:

$$
J_{2}=\int_{\Omega}(\nabla \mathbf{u} \cdot \nabla) \mathbf{d} \cdot \nabla(\Delta \widehat{\mathbf{d}}) d \Omega+\int_{\Omega}(\mathbf{u} \cdot \nabla) \nabla \mathbf{d} \cdot \nabla(\Delta \widehat{\mathbf{d}}) d \Omega:=K_{1}+K_{2}
$$

The estimate of the term $K_{1}$ is similar to (14), (15) and (16) (again, this estimates are valid only for the cases $2 \leq q \leq 3)$ :

$$
K_{1} \leq \bar{\varepsilon}\|\nabla(\Delta \widehat{\mathbf{d}})\|_{L^{2}(\Omega)}^{2}+C_{\bar{\varepsilon}}\|\nabla \mathbf{u}\|_{L^{q}(\Omega)}^{\frac{2 q}{2-3}}\left(\|\Delta \widehat{\mathbf{d}}\|_{L^{2}(\Omega)}^{2}+\|\widetilde{\mathbf{d}}\|_{H^{2}(\Omega)}^{2}\right)
$$


The estimate for $K_{2}$ is the following for $2 \leq q<3$ (in fact, the argument is valid for $3 / 2<q<3):$

$$
K_{2} \leq \bar{\varepsilon}\|\nabla(\Delta \widehat{\mathbf{d}})\|_{L^{2}(\Omega)}^{2}+C_{\bar{\varepsilon}} \int_{\Omega}|\mathbf{u}|^{2}\left|D^{2} \mathbf{d}\right|^{2} d \Omega
$$

and the last term on the right hand side is bounded as follows:

$$
\begin{aligned}
\int_{\Omega}|\mathbf{u}|^{2}\left|D^{2} \mathbf{d}\right|^{2} d \Omega & \leq\|\mathbf{u}\|_{L^{\frac{q}{3-q}(\Omega)}}^{2}\left\|D^{2} \mathbf{d}\right\|_{L^{\frac{6 q}{5 q-6}(\Omega)}}^{2} \quad(q<3) \\
& \leq C\|\nabla \mathbf{u}\|_{L^{q}(\Omega)}^{2}\left\|D^{2} \mathbf{d}\right\|_{L^{2}(\Omega)}^{\frac{2(2 q-3)}{q}}\left\|D^{2} \mathbf{d}\right\|_{H^{1}(\Omega)}^{\frac{2(3-q)}{q}} \\
& \leq \bar{\varepsilon}\left\|D^{2} \widehat{\mathbf{d}}\right\|_{H^{1}(\Omega)}^{2}+C_{\bar{\varepsilon}}\|\nabla \mathbf{u}\|_{L^{q}(\Omega)}^{\frac{2 q}{2 q-3}}\left(\left\|D^{2} \widehat{\mathbf{d}}\right\|_{L^{2}(\Omega)}^{2}+\left\|D^{2} \widetilde{\mathbf{d}}\right\|_{L^{2}(\Omega)}^{2}\right) \\
& +C\left\|D^{2} \widetilde{\mathbf{d}}\right\|_{H^{1}(\Omega)}^{2}
\end{aligned}
$$

In order to obtain a bound of $K_{2}$ for $q=3$, we consider the following decomposition of $K_{2}$ :

$$
K_{2}=-\int_{\Omega}(\nabla \mathbf{u} \cdot \nabla) \nabla \mathbf{d} \cdot \Delta \widehat{\mathbf{d}} d \Omega-\int_{\Omega}(\mathbf{u} \cdot \nabla) \Delta \widetilde{\mathbf{d}} \Delta \widehat{\mathbf{d}} d \Omega:=K_{21}+K_{22}
$$

(where the equality $\int_{\Omega}(\mathbf{u} \cdot \nabla) \Delta \widehat{\mathbf{d}} \cdot \Delta \widehat{\mathbf{d}} d \Omega=0$ has been used), where:

$$
\begin{aligned}
K_{21} & \leq\|\nabla \mathbf{u}\|_{L^{3}(\Omega)}\left\|D^{2} \mathbf{d}\right\|_{L^{3}(\Omega)}\left\|D^{2} \widehat{\mathbf{d}}\right\|_{L^{3}(\Omega)} \\
& \leq C\|\nabla \mathbf{u}\|_{L^{3}(\Omega)}\left\|D^{2} \mathbf{d}\right\|_{L^{2}(\Omega)}^{1 / 2}\left\|D^{2} \mathbf{d}\right\|_{H^{1}(\Omega)}^{1 / 2}\left\|D^{2} \widehat{\mathbf{d}}\right\|_{L^{2}(\Omega)}^{1 / 2}\left\|D^{2} \widehat{\mathbf{d}}\right\|_{H^{1}(\Omega)}^{1 / 2} \\
& \leq C\|\nabla \mathbf{u}\|_{L^{3}(\Omega)}\left\{\|\Delta \widehat{\mathbf{d}}\|_{L^{2}(\Omega)}\|\Delta \widehat{\mathbf{d}}\|_{H^{1}(\Omega)}+\|\Delta \widehat{\mathbf{d}}\|_{L^{2}(\Omega)}^{1 / 2}\|\Delta \widehat{\mathbf{d}}\|_{H^{1}(\Omega)}^{1 / 2}\|\widetilde{\mathbf{d}}\|_{H^{2}(\Omega)}^{1 / 2}\|\widetilde{\mathbf{d}}\|_{H^{3}(\Omega)}^{1 / 2}\right\} \\
& \leq \bar{\varepsilon}\|\Delta \widehat{\mathbf{d}}\|_{H^{1}(\Omega)}^{2}+C_{\bar{\varepsilon}}\|\nabla \mathbf{u}\|_{L^{3}(\Omega)}^{2}\left(\|\Delta \widehat{\mathbf{d}}\|_{L^{2}(\Omega)}^{2}+\|\widetilde{\mathbf{d}}\|_{H^{2}(\Omega)}^{2}\right)+C\|\widetilde{\mathbf{d}}\|_{H^{3}(\Omega)}^{2}
\end{aligned}
$$

In order to bound $K_{22}$, integrating by parts, we get:

$$
K_{22}=\int_{\Omega}(\mathbf{u} \cdot \nabla) \Delta \widehat{\mathbf{d}} \Delta \widetilde{\mathbf{d}} d \Omega \leq \bar{\varepsilon}\|\nabla(\Delta \widehat{\mathbf{d}})\|_{L^{2}(\Omega)}^{2}+C_{\bar{\varepsilon}} \int_{\Omega}|\mathbf{u}|^{2}|\Delta \widetilde{\mathbf{d}}|^{2} d \Omega
$$


From hypothesis $\nabla \mathbf{u} \in L^{2}\left(0, T ; \mathbf{L}^{3}(\Omega)\right)$, in particular $\mathbf{u} \in L^{2}\left(0, T ; \mathbf{L}^{p}(\Omega)\right), \forall p<+\infty$. Then,

$$
\begin{aligned}
\int_{\Omega}|\mathbf{u}|^{2}|\Delta \widetilde{\mathbf{d}}|^{2} d \Omega & \leq C\|\mathbf{u}\|_{L^{p}(\Omega)}^{2}\|\Delta \widetilde{\mathbf{d}}\|_{L^{2+\delta(p)}}^{2} \\
& \leq C\|\nabla \mathbf{u}\|_{L^{3}(\Omega)}^{2}\|\Delta \widetilde{\mathbf{d}}\|_{L^{2+\delta(p)}(\Omega)}^{2} \quad \text { for a certain } \delta(p)>0
\end{aligned}
$$

and therefore, the previous bounds belongs to $L^{1}(0, T)$ imposing the following additional regularity $\Delta \widetilde{\mathbf{d}} \in L^{\infty}\left(0, T ; \mathbf{L}^{2+\delta}(\Omega)\right)$ for some $\delta>0(\delta=\delta(p) \downarrow 0$ as $p \uparrow+\infty)$, that is,

$$
\widetilde{\mathbf{d}} \in L^{\infty}\left(0, T ; \mathbf{W}^{2, s}(\Omega)\right), \quad \text { for some } s>2 .
$$

Collecting all the previous estimates in (20) and adding $\gamma\|\Delta \widehat{\mathbf{d}}\|_{L^{2}(\Omega)}^{2}$ in both sides of (20), we obtain:

- for $q \in[2,3)$,

$$
\begin{aligned}
\frac{d}{d t}\|\Delta \widehat{\mathbf{d}}\|_{L^{2}(\Omega)}^{2} & +\gamma\|\widehat{\mathbf{d}}\|_{H^{3}(\Omega)}^{2} \leq C\left\{\|\widetilde{\mathbf{d}}\|_{H^{3}(\Omega)}^{2}+\|\widehat{\mathbf{d}}\|_{H^{1}(\Omega)}^{2}\right. \\
& \left.+\|\nabla \mathbf{u}\|_{L^{q}(\Omega)}^{\frac{2 q}{2 q-3}}\left(\|\Delta \widehat{\mathbf{d}}\|_{L^{2}(\Omega)}^{2}+\|\widetilde{\mathbf{d}}\|_{H^{2}(\Omega)}^{2}\right)\right\}
\end{aligned}
$$

- for $q=3$,

$$
\begin{aligned}
\frac{d}{d t}\|\Delta \widehat{\mathbf{d}}\|_{L^{2}(\Omega)}^{2} & +\gamma\|\widehat{\mathbf{d}}\|_{H^{3}(\Omega)}^{2} \leq C\left\{\|\widetilde{\mathbf{d}}\|_{H^{3}(\Omega)}^{2}+\|\widehat{\mathbf{d}}\|_{H^{1}(\Omega)}^{2}\right. \\
& \left.+\|\nabla \mathbf{u}\|_{L^{3}(\Omega)}^{2}\left(\|\Delta \widehat{\mathbf{d}}\|_{L^{2}(\Omega)}^{2}+\|\widetilde{\mathbf{d}}\|_{H^{2}(\Omega)}^{2}+\|\Delta \widetilde{\mathbf{d}}\|_{L^{2+\delta}(\Omega)}^{2}\right)\right\}
\end{aligned}
$$

Therefore, applying the Gronwall's Lemma we obtain that:

$$
\widehat{\mathbf{d}} \in L^{\infty}\left(0, T ; \mathbf{H}^{2}(\Omega)\right) \cap L^{2}\left(0, T ; \mathbf{H}^{3}(\Omega)\right) .
$$

In order to obtain the strong regularity for $\mathbf{u}$, that is, $\mathbf{u} \in L^{\infty}\left(0, T ; \mathbf{H}^{1}(\Omega)\right) \cap L^{2}\left(0, T ; \mathbf{H}^{2}(\Omega)\right)$, there are not differences with the homogeneous Neumann boundary condition case (see Lemma 2.2). Neither the obtention of regularity for $\partial_{t} \mathbf{u}$ neither $\partial_{t} \mathbf{d}$ are different (see Lemma 2.3). Thus, as in the Neumann case, we can conclude that $\partial_{t} \mathbf{u} \in L^{2}\left(0, T ; \mathbf{L}^{2}(\Omega)\right)$, $\partial_{t} \mathbf{d} \in L^{\infty}\left(0, T ; \mathbf{L}^{2}(\Omega)\right) \cap L^{2}\left(0, T ; \mathbf{H}^{1}(\Omega)\right)$ and $p \in L^{2}\left(0, T ; H^{1}(\Omega)\right)$. 


\section{Proof of Theorem 1.2 (when (H1) is imposed).}

We want to analyze those cases from the precedent Section where it could not be deduced the strong regularity for $\mathbf{d}$. For this aim, we will use Ladyzhenskaya's type of estimates. We study again separately the homogeneous Neumann boundary conditions for $\mathbf{d}$, i. e., $\left.\partial_{\mathbf{n}} \mathbf{d}\right|_{\partial \Omega}=\mathbf{0}$, and the non-homogeneous Dirichlet boundary conditions, that is, $\left.\mathbf{d}\right|_{\partial \Omega}=\mathbf{h}$. The periodic boundary conditions for $\mathbf{d}$ will be treated in the next Section.

\subsection{Homogeneous Neumann boundary conditions}

Remember that we use the following boundary condition for $\mathbf{d}$ :

$$
\partial_{\mathbf{n}} \mathbf{d}=\mathbf{0} \quad \text { on } \Sigma
$$

Deriving $(L C)$ with respect to the time, and taking first $\partial_{t} \mathbf{d}$ as test function for $(L C)_{3}$, we obtain (using $\left.\left|\partial_{t} \mathbf{f}_{\delta}(\mathbf{d})\right| \leq C\left|\partial_{t} \mathbf{d}\right|\right)$ :

$$
\frac{1}{2} \frac{d}{d t}\left\|\partial_{t} \mathbf{d}\right\|_{L^{2}(\Omega)}^{2}+\left\|\nabla\left(\partial_{t} \mathbf{d}\right)\right\|_{L^{2}(\Omega)}^{2} \leq C\left(\left\|\partial_{t} \mathbf{u}\right\|_{L^{2}(\Omega)}^{2}+\left\|\partial_{t} \mathbf{d}\right\|_{L^{2}(\Omega)}^{2}\right)
$$

(where we have integrated by parts the term $\int_{\Omega}\left(\partial_{t} \mathbf{u} \cdot \nabla\right) \mathbf{d} \cdot \partial_{t} \mathbf{d} d \Omega$ ). On the other hand, taking $\lambda^{-1} \partial_{t} \mathbf{u}$ and $-\Delta\left(\partial_{t} \mathbf{d}\right)$ as test functions, we obtain:

$$
\begin{aligned}
& \frac{1}{2} \frac{d}{d t}\left(\lambda^{-1}\left\|\partial_{t} \mathbf{u}\right\|_{L^{2}(\Omega)}^{2}+\left\|\nabla\left(\partial_{t} \mathbf{d}\right)\right\|_{L^{2}(\Omega)}^{2}\right)+\frac{\nu}{\lambda}\left\|\nabla\left(\partial_{t} \mathbf{u}\right)\right\|_{L^{2}(\Omega)}^{2}+\gamma\left\|\Delta\left(\partial_{t} \mathbf{d}\right)\right\|_{L^{2}(\Omega)}^{2} \\
& \quad \leq \int_{\Omega}\left(\partial_{t} \mathbf{f}_{\delta}(\mathbf{d}) \cdot \partial_{t}(\Delta \mathbf{d})+(\mathbf{u} \cdot \nabla)\left(\partial_{t} \mathbf{d}\right) \cdot \partial_{t}(\Delta \mathbf{d})-\frac{1}{\lambda}\left(\partial_{t} \mathbf{u} \cdot \nabla\right) \mathbf{u} \cdot\left(\partial_{t} \mathbf{u}\right)\right) d \Omega \\
& \quad-\int_{\Omega}\left(\nabla\left(\partial_{t} \mathbf{d}\right)\right)^{t} \cdot \Delta \mathbf{d} \cdot \partial_{t} \mathbf{u} d \Omega \\
& \leq C\left\{\int_{\Omega}\left|\partial_{t} \mathbf{d}\left\|\Delta\left(\partial_{t} \mathbf{d}\right)\left|+\int_{\Omega}\right| \nabla \mathbf{u}\right\| \nabla\left(\partial_{t} \mathbf{d}\right)\right|^{2} d \Omega+\lambda^{-1} \int_{\Omega}\left|\partial_{t} \mathbf{u}\right|^{2}|\nabla \mathbf{u}| d \Omega\right\} \\
& -\int_{\Omega}\left(\nabla\left(\partial_{t} \mathbf{d}\right)\right)^{t} \cdot \Delta \mathbf{d} \cdot \partial_{t} \mathbf{u} d \Omega=\sum_{i=1}^{4} J_{i} .
\end{aligned}
$$

In fact, we proceed as follows:

$$
J_{1} \leq C\left\|\partial_{t} \mathbf{d}\right\|_{L^{2}(\Omega)}\left\|\Delta\left(\partial_{t} \mathbf{d}\right)\right\|_{L^{2}(\Omega)} \leq \bar{\varepsilon}\left\|\nabla\left(\partial_{t} \mathbf{d}\right)\right\|_{H^{1}(\Omega)}^{2}+C_{\bar{\varepsilon}}\left\|\partial_{t} \mathbf{d}\right\|_{L^{2}(\Omega)}^{2}
$$


The estimate for $J_{2}$ is similar to (13):

$$
\begin{aligned}
J_{2} & \leq\|\nabla \mathbf{u}\|_{L^{q}(\Omega)}\left\|\nabla\left(\partial_{t} \mathbf{d}\right)\right\|_{L^{\frac{2 q}{q-1}(\Omega)}}^{2} \leq\|\nabla \mathbf{u}\|_{L^{q}(\Omega)}\left\|\nabla\left(\partial_{t} \mathbf{d}\right)\right\|_{L^{2}(\Omega)}^{\frac{2 q-3}{q}}\left\|\nabla\left(\partial_{t} \mathbf{d}\right)\right\|_{H^{1}(\Omega)}^{\frac{3}{q}} \\
& \leq \bar{\varepsilon}\left\|\nabla\left(\partial_{t} \mathbf{d}\right)\right\|_{H^{1}(\Omega)}^{2}+C_{\bar{\varepsilon}}\|\nabla \mathbf{u}\|_{L^{q}(\Omega)}^{\frac{2 q}{2-3}}\left\|\nabla\left(\partial_{t} \mathbf{d}\right)\right\|_{L^{2}(\Omega)}^{2},
\end{aligned}
$$

where the additional regularity $(H 1)$ for $\nabla \mathbf{u}$ appears. The term $J_{3}$ can be bound as in Berselli's article [3]:

$$
\begin{aligned}
J_{3} & \leq \lambda^{-1}\left\|\partial_{t} \mathbf{u}\right\|_{L^{\frac{2 q}{q-1}(\Omega)}}^{2}\|\nabla \mathbf{u}\|_{L^{q}(\Omega)} \leq C\left\|\partial_{t} \mathbf{u}\right\|_{L^{2}(\Omega)}^{\frac{2 q-3}{q}}\left\|\nabla\left(\partial_{t} \mathbf{u}\right)\right\|_{L^{2}(\Omega)}^{\frac{3}{q}}\|\nabla \mathbf{u}\|_{L^{q}(\Omega)} \\
& \leq \bar{\varepsilon}\left\|\nabla\left(\partial_{t} \mathbf{u}\right)\right\|_{L^{2}(\Omega)}^{2}+C_{\bar{\varepsilon}}\|\nabla \mathbf{u}\|_{L^{q}(\Omega)}^{\frac{2 q}{2 q-3}}\left\|\partial_{t} \mathbf{u}\right\|_{L^{2}(\Omega)}^{2}
\end{aligned}
$$

Summary, for $\bar{\varepsilon}$ small enough, we have the following inequality:

$$
\begin{aligned}
& \frac{d}{d t}\left(\lambda^{-1}\left\|\partial_{t} \mathbf{u}\right\|_{L^{2}(\Omega)}^{2}+\left\|\partial_{t} \mathbf{d}\right\|_{H^{1}(\Omega)}^{2}\right)+\frac{\nu}{\lambda}\left\|\partial_{t} \mathbf{u}\right\|_{H^{1}(\Omega)}^{2}+\gamma\left\|\partial_{t} \mathbf{d}\right\|_{H^{2}(\Omega)}^{2} \\
& \quad \leq C\left(\|\nabla \mathbf{u}\|_{L^{q}(\Omega)}^{\frac{2 q}{2 q-3}}+1\right)\left(\left\|\partial_{t} \mathbf{u}\right\|_{L^{2}(\Omega)}^{2}+\left\|\partial_{t} \mathbf{d}\right\|_{H^{1}(\Omega)}^{2}\right) \\
& \quad-\int_{\Omega}\left(\partial_{t} \mathbf{u} \cdot \nabla\right)\left(\partial_{t} \mathbf{d}\right) \cdot \Delta \mathbf{d} d \Omega
\end{aligned}
$$

Finally, there is left the last term to bound. We can choose two possible strategies, depending on the way we write such term:

1. If we try to integrate the term directly, we need to impose the additional regularity (H4) for $\Delta \mathbf{d}$, estimating as follows:

$$
\begin{aligned}
& \int_{\Omega}\left|\nabla \partial_{t} \mathbf{d}^{t}\|\Delta \mathbf{d}\| \partial_{t} \mathbf{u}\right| d \Omega \leq\left\|\partial_{t} \mathbf{u}\right\|_{L^{\frac{2 q}{q-1}(\Omega)}}\|\Delta \mathbf{d}\|_{L^{q}(\Omega)}\left\|\nabla\left(\partial_{t} \mathbf{d}\right)\right\|_{L^{\frac{2 q}{q-1}(\Omega)}} \\
& \quad \leq\left\|\partial_{t} \mathbf{u}\right\|_{L^{2}(\Omega)}^{\alpha}\left\|\nabla\left(\partial_{t} \mathbf{u}\right)\right\|_{L^{2}(\Omega)}^{1-\alpha}\|\Delta \mathbf{d}\|_{L^{q}(\Omega)}\left\|\nabla\left(\partial_{t} \mathbf{d}\right)\right\|_{L^{2}(\Omega)}^{\alpha}\left\|\nabla\left(\partial_{t} \mathbf{d}\right)\right\|_{H^{1}(\Omega)}^{1-\alpha} \\
& \quad \leq \bar{\varepsilon}\left(\left\|\nabla\left(\partial_{t} \mathbf{u}\right)\right\|_{L^{2}(\Omega)}^{2}+\left\|\nabla\left(\partial_{t} \mathbf{d}\right)\right\|_{H^{1}(\Omega)}^{2}\right)+C_{\bar{\varepsilon}}\|\Delta \mathbf{d}\|_{L^{q}(\Omega)}^{1 / \alpha}\left(\left\|\partial_{t} \mathbf{u}\right\|_{L^{2}(\Omega)}^{2}+\left\|\nabla\left(\partial_{t} \mathbf{d}\right)\right\|_{L^{2}(\Omega)}^{2}\right)
\end{aligned}
$$

with $\frac{1}{\alpha}=\frac{2 q}{2 q-3}$. We can conclude the following inequality:

$$
\begin{aligned}
& \frac{d}{d t}\left(\lambda^{-1}\left\|\partial_{t} \mathbf{u}\right\|_{L^{2}(\Omega)}^{2}+\left\|\partial_{t} \mathbf{d}\right\|_{H^{1}(\Omega)}^{2}\right)+\frac{\nu}{\lambda}\left\|\partial_{t} \mathbf{u}\right\|_{H^{1}(\Omega)}^{2}+\gamma\left\|\partial_{t} \mathbf{d}\right\|_{H^{2}(\Omega)}^{2} \\
& \leq C\left\{\left(\|\nabla \mathbf{u}\|_{L^{q^{2}(\Omega)}}^{\frac{2 q}{22-3}}+\|\Delta \mathbf{d}\|_{L^{q}(\Omega)}^{\frac{2 q}{2 q-3}}+1\right)\left(\left\|\partial_{t} \mathbf{u}\right\|_{L^{2}(\Omega)}^{2}+\left\|\partial_{t} \mathbf{d}\right\|_{H^{1}(\Omega)}^{2}\right)\right\}
\end{aligned}
$$


2. If we integrate by parts, we will have to impose the hypothesis (H3) on $\nabla \mathbf{d}$. Indeed,

$$
\begin{aligned}
-\int_{\Omega}\left(\partial_{t} \mathbf{u} \cdot \nabla\right)\left(\partial_{t} \mathbf{d}\right) \cdot \Delta \mathbf{d} d \Omega & =\int_{\Omega}\left(\partial_{t} \mathbf{u} \cdot \nabla\right) \nabla\left(\partial_{t} \mathbf{d}\right) \cdot \nabla \mathbf{d} d \Omega \\
& +\int_{\Omega}\left(\nabla\left(\partial_{t} \mathbf{u}\right) \cdot \nabla\right)\left(\partial_{t} \mathbf{d}\right): \nabla \mathbf{d} d \Omega
\end{aligned}
$$

because of the boundary term vanishes due to $\left.\partial_{t} \mathbf{u}\right|_{\partial \Omega}=\mathbf{0}$, independently from the boundary conditions imposed on $\mathbf{d}$. The remaining terms can be bounded as follows:

$$
\begin{gathered}
\int_{\Omega}\left|D^{2}\left(\partial_{t} \mathbf{d}\right)\|\nabla \mathbf{d}\| \partial_{t} \mathbf{u}\right| d \Omega \leq\left\|D^{2}\left(\partial_{t} \mathbf{d}\right)\right\|_{L^{2}(\Omega)}\|\nabla \mathbf{d}\|_{L^{p}(\Omega)}\left\|\partial_{t} \mathbf{u}\right\|_{L^{\frac{2 p}{p-2}}(\Omega)} \\
\leq\left\|\partial_{t} \mathbf{d}\right\|_{H^{2}(\Omega)}\|\nabla \mathbf{d}\|_{L^{p}(\Omega)}\left\|\partial_{t} \mathbf{u}\right\|_{L^{2}(\Omega)}^{\frac{p-3}{p}}\left\|\nabla\left(\partial_{t} \mathbf{u}\right)\right\|_{L^{2}(\Omega)}^{\frac{3}{p}} \\
\leq \bar{\varepsilon}\left(\left\|\partial_{t} \mathbf{d}\right\|_{H^{2}(\Omega)}^{2}+\left\|\partial_{t} \mathbf{u}\right\|_{H^{1}(\Omega)}^{2}\right)+C_{\bar{\varepsilon}}\|\nabla \mathbf{d}\|_{L^{p}(\Omega)}^{\frac{2 p}{p-3}}\left\|\partial_{t} \mathbf{u}\right\|_{L^{2}(\Omega)}^{2}
\end{gathered}
$$

And similarly,

$$
\begin{gathered}
\int_{\Omega}\left|\nabla\left(\partial_{t} \mathbf{d}\right)\right||\nabla \mathbf{d}|\left|\nabla\left(\partial_{t} \mathbf{u}\right)\right| d \Omega \leq\left\|\nabla\left(\partial_{t} \mathbf{d}\right)\right\|_{L^{\frac{2 p}{p-2}(\Omega)}}\|\nabla \mathbf{d}\|_{L^{p}(\Omega)}\left\|\nabla\left(\partial_{t} \mathbf{u}\right)\right\|_{L^{2}(\Omega)} \\
\leq\left\|\nabla\left(\partial_{t} \mathbf{d}\right)\right\|_{L^{2}(\Omega)}^{\frac{p-3}{p}}\left\|\nabla\left(\partial_{t} \mathbf{d}\right)\right\|_{H^{1}(\Omega)}^{\frac{3}{p}}\|\nabla \mathbf{d}\|_{L^{p}(\Omega)}\left\|\partial_{t} \mathbf{u}\right\|_{H^{1}(\Omega)} \\
\leq \bar{\varepsilon}\left(\left\|\partial_{t} \mathbf{d}\right\|_{H^{2}(\Omega)}^{2}+\left\|\partial_{t} \mathbf{u}\right\|_{H^{1}(\Omega)}^{2}\right)+C_{\bar{\varepsilon}}\|\nabla \mathbf{d}\|_{L^{p}(\Omega)}^{\frac{2 p}{p-3}}\left\|\nabla\left(\partial_{t} \mathbf{d}\right)\right\|_{L^{2}(\Omega)}^{2}
\end{gathered}
$$

Therefore, we obtain the following inequality:

$$
\begin{aligned}
& \frac{d}{d t}\left(\lambda^{-1}\left\|\partial_{t} \mathbf{u}\right\|_{L^{2}(\Omega)}^{2}+\left\|\partial_{t} \mathbf{d}\right\|_{H^{1}(\Omega)}^{2}\right)+\frac{\nu}{\lambda}\left\|\partial_{t} \mathbf{u}\right\|_{H^{1}(\Omega)}^{2}+\gamma\left\|\partial_{t} \mathbf{d}\right\|_{H^{2}(\Omega)}^{2} \\
& \quad \leq C\left(\|\nabla \mathbf{d}\|_{L^{p}(\Omega)}^{\frac{2 p}{p-3}}+\|\nabla \mathbf{u}\|_{L^{q}(\Omega)}^{\frac{2 q}{2 q-3}}+1\right)\left(\left\|\partial_{t} \mathbf{u}\right\|_{L^{2}(\Omega)}^{2}+\left\|\partial_{t} \mathbf{d}\right\|_{H^{1}(\Omega)}^{2}\right)
\end{aligned}
$$

Thus, using the Gronwall's Lemma, we can conclude (as in [3]) that:

$$
\begin{aligned}
& \partial_{t} \mathbf{u} \in L^{\infty}\left(\varepsilon, T ; \mathbf{L}^{2}(\Omega)\right) \cap L^{2}\left(\varepsilon, T ; \mathbf{H}^{1}(\Omega)\right), \\
& \partial_{t} \mathbf{d} \in L^{\infty}\left(\varepsilon, T ; \mathbf{H}^{1}(\Omega)\right) \cap L^{2}\left(\varepsilon, T ; \mathbf{H}^{2}(\Omega)\right)
\end{aligned}
$$

In particular, from $\mathbf{u}$ and $\partial_{t} \mathbf{u} \in L^{2}\left(\varepsilon, T ; \mathbf{H}^{1}(\Omega)\right)$, we obtain $\mathbf{u} \in C\left([\varepsilon, T] ; \mathbf{H}^{1}(\Omega)\right)(\mathbf{d}$ and $\partial_{t} \mathbf{d} \in L^{2}\left(\varepsilon, T ; \mathbf{H}^{2}(\Omega)\right)$ implies that $\mathbf{d} \in C\left([\varepsilon, T] ; \mathbf{H}^{2}(\Omega)\right)$. Therefore, using the local in time strong regularity as in [3], we can conclude that:

$$
\nabla \mathbf{u} \in L^{\infty}\left(0, T ; \mathbf{L}^{2}(\Omega)\right) \quad \text { y } \quad \Delta \mathbf{d} \in L^{\infty}\left(0, T ; \mathbf{L}^{2}(\Omega)\right) .
$$


Then, $\mathbf{d} \in L^{\infty}\left(0, T ; \mathbf{H}^{2}(\Omega)\right)$ and $\mathbf{u} \in L^{\infty}\left(0, T ; \mathbf{H}^{1}(\Omega)\right)$.

Thus, it is only necessary to obtain $\mathbf{d} \in L^{2}\left(0, T ; \mathbf{H}^{3}(\Omega)\right)$ and $\mathbf{u} \in L^{2}\left(0, T ; \mathbf{H}^{2}(\Omega)\right)$. To this aim, we use the following result:

Lemma 3.1 Suppose that $\mathbf{d} \in L^{\infty}\left(0, T ; \mathbf{H}^{2}(\Omega)\right)$ and $\mathbf{u} \in L^{\infty}\left(0, T ; \mathbf{H}^{1}(\Omega)\right)$, then $\mathbf{d} \in$ $L^{2}\left(0, T ; \mathbf{H}^{3}(\Omega)\right)$ and $\mathbf{u} \in L^{2}\left(0, T ; \mathbf{H}^{2}(\Omega)\right)$. Moreover, $\partial_{t} \mathbf{d} \in L^{\infty}\left(0, T ; \mathbf{L}^{2}(\Omega)\right) \cap L^{2}\left(0, T ; \mathbf{H}^{1}(\Omega)\right)$, $\partial_{t} \mathbf{u} \in L^{2}\left(0, T ; \mathbf{L}^{2}(\Omega)\right)$ and $p \in L^{2}\left(0, T ; H^{1}(\Omega)\right)$.

Proof: See Appendix A.4.

\subsection{Nonhomogeneous Dirichlet boundary condition case}

In this case, we derive the equation for $\mathbf{u}$ in $(L C)$ and the system for $\widehat{\mathbf{d}}(19)$ with respect to the time, and take $\lambda^{-1} \partial_{t} \mathbf{u}$ and $-\Delta\left(\partial_{t} \widehat{\mathbf{d}}\right)$, respectively, as test functions. Thus we obtain an inequality similar to (25) (with three additional terms (on the right)). Concretely:

$$
\begin{aligned}
& \frac{1}{2} \frac{d}{d t}\left(\lambda^{-1}\left\|\partial_{t} \mathbf{u}\right\|_{L^{2}(\Omega)}^{2}+\left\|\partial_{t} \widehat{\mathbf{d}}\right\|_{H^{1}(\Omega)}^{2}\right)+\frac{\nu}{\lambda}\left\|\partial_{t} \mathbf{u}\right\|_{H^{1}(\Omega)}^{2}+\gamma\left\|\partial_{t} \widehat{\mathbf{d}}\right\|_{H^{2}(\Omega)}^{2} \\
& \quad \leq \bar{\varepsilon}\left\|\Delta\left(\partial_{t} \widehat{\mathbf{d}}\right)\right\|_{L^{2}(\Omega)}^{2}+C_{\bar{\varepsilon}} \int_{\Omega}\left(\left|\partial_{t} \widehat{\mathbf{d}}\right|^{2}+\left|\partial_{t} \widetilde{\mathbf{d}}\right|^{2}\right) d \Omega+\lambda^{-1} \int_{\Omega}\left|\partial_{t} \mathbf{u}\right|^{2}|\nabla \mathbf{u}| d \Omega \\
& \quad-\int_{\Omega}\left(\partial_{t} \mathbf{u} \cdot \nabla\right) \partial_{t} \mathbf{d} \cdot \Delta \mathbf{d} d \Omega-\int_{\Omega}\left(\partial_{t} \mathbf{u} \cdot \nabla\right) \mathbf{d} \cdot \Delta\left(\partial_{t} \mathbf{d}\right) d \Omega \\
& \quad+\int_{\Omega}\left(\partial_{t} \mathbf{u} \cdot \nabla\right) \mathbf{d} \cdot \Delta\left(\partial_{t} \widehat{\mathbf{d}}\right) d \Omega+\int_{\Omega}(\mathbf{u} \cdot \nabla)\left(\partial_{t} \mathbf{d}\right) \cdot \Delta\left(\partial_{t} \widehat{\mathbf{d}}\right) d \Omega:=\sum_{i=1}^{7} \widetilde{J}_{i}
\end{aligned}
$$

The estimate of $\widetilde{J}_{3}$ is identical to those of $J_{3}$ (in the Neumann case).

The term $\widetilde{J}_{4}$ can be bounded in two different ways (as the $J_{4}$-term in the homogeneous Neumann boundary case), depending on te hypothesis imposed on $\mathbf{d}$. That is:

- if the hypothesis $(H 4)$ on $\Delta \mathbf{d}$ is considered, then:

$$
\begin{aligned}
\widetilde{J}_{4} & \leq \bar{\varepsilon}\left(\left\|\partial_{t} \mathbf{u}\right\|_{H^{1}(\Omega)}^{2}+\left\|\partial_{t} \widehat{\mathbf{d}}\right\|_{H^{2}(\Omega)}^{2}+\left\|\partial_{t} \widetilde{\mathbf{d}}\right\|_{H^{2}(\Omega)}^{2}\right) \\
& +C_{\bar{\varepsilon}}\|\Delta \mathbf{d}\|_{L^{q}(\Omega)}^{\frac{2 q}{2 q-3}}\left(\left\|\partial_{t} \mathbf{u}\right\|_{L^{2}(\Omega)}^{2}+\left\|\partial_{t} \widehat{\mathbf{d}}\right\|_{H^{1}(\Omega)}^{2}+\left\|\partial_{t} \widetilde{\mathbf{d}}\right\|_{H^{1}(\Omega)}^{2}\right)
\end{aligned}
$$


- if the hypothesis (H3) on $\nabla \mathbf{d}$ is considered, then:

$$
\begin{aligned}
\widetilde{J}_{4} & \leq \bar{\varepsilon}\left(\left\|\partial_{t} \mathbf{u}\right\|_{H^{1}(\Omega)}^{2}+\left\|\partial_{t} \widehat{\mathbf{d}}\right\|_{H^{2}(\Omega)}^{2}+\left\|\partial_{t} \widetilde{\mathbf{d}}\right\|_{H^{2}(\Omega)}^{2}\right) \\
& +C_{\bar{\varepsilon}}\|\nabla \mathbf{d}\|_{L^{p}(\Omega)}^{\frac{2 p}{p-3}}\left(\left\|\partial_{t} \mathbf{u}\right\|_{L^{2}(\Omega)}^{2}+\left\|\partial_{t} \widehat{\mathbf{d}}\right\|_{H^{1}(\Omega)}^{2}+\left\|\partial_{t} \widetilde{\mathbf{d}}\right\|_{H^{1}(\Omega)}^{2}\right)
\end{aligned}
$$

Adding $\widetilde{J}_{5}$ and $\widetilde{J}_{6}$ :

$$
\widetilde{J}_{5}+\widetilde{J}_{6}=-\int_{\Omega}\left(\partial_{t} \mathbf{u} \cdot \nabla\right) \mathbf{d} \cdot \Delta\left(\partial_{t} \widetilde{\mathbf{d}}\right) d \Omega
$$

If we consider $(H 3)$ on $\nabla \mathbf{d}$, we bound as follows:

$$
\widetilde{J}_{5}+\widetilde{J}_{6} \leq \bar{\varepsilon}\left(\left\|\partial_{t} \mathbf{u}\right\|_{H^{1}(\Omega)}^{2}+\left\|\partial_{t} \widetilde{\mathbf{d}}\right\|_{H^{2}(\Omega)}^{2}\right)+C_{\bar{\varepsilon}}\|\nabla \mathbf{d}\|_{L^{p}(\Omega)}^{\frac{2 p}{p-3}}\left\|\partial_{t} \mathbf{u}\right\|_{L^{2}(\Omega)}^{2}
$$

If we consider $(H 4)$ hypothesis for $\Delta \mathbf{d}$, we can bound as follows:

$$
\begin{aligned}
\widetilde{J}_{5}+\widetilde{J}_{6} & \leq\left\|\partial_{t} \mathbf{u}\right\|_{L^{\frac{2 p}{p-2}(\Omega)}}\|\nabla \mathbf{d}\|_{L^{p}(\Omega)}\left\|\Delta\left(\partial_{t} \widetilde{\mathbf{d}}\right)\right\|_{L^{2}(\Omega)} \\
& \leq C\left\|\partial_{t} \mathbf{u}\right\|_{L^{2}(\Omega)}^{\beta}\left\|\partial_{t} \mathbf{u}\right\|_{H^{1}(\Omega)}^{1-\beta}\left(\|\Delta \mathbf{d}\|_{L^{q}(\Omega)}+\|\mathbf{d}\|_{L^{q}(\Omega)}\right)\left\|\partial_{t} \widetilde{\mathbf{d}}\right\|_{H^{2}(\Omega)} \\
& \leq \bar{\varepsilon}\left\|\partial_{t} \mathbf{u}\right\|_{H^{1}(\Omega)}^{2}+C_{\bar{\varepsilon}}\left\{\left\|\partial_{t} \widetilde{\mathbf{d}}\right\|_{H^{2}(\Omega)}^{2}+\left(\|\Delta \mathbf{d}\|_{L^{q}(\Omega)}^{\frac{2}{\beta}}+\|\mathbf{d}\|_{H^{1}(\Omega)}^{\frac{2}{\beta}}\right)\left\|\partial_{t} \mathbf{u}\right\|_{L^{2}(\Omega)}\right\}
\end{aligned}
$$

where $p=\frac{3 q}{3-q}, \frac{2}{\beta}=\frac{2 p}{p-3}=\frac{2 q}{2 q-3}$ if $q<3$. For $q>3$, we integrate by parts and:

$$
\widetilde{J}_{5}+\widetilde{J}_{6} \approx \int_{\Omega}\left|\partial_{t} \mathbf{u}\right|\left|\nabla\left(\partial_{t} \widetilde{\mathbf{d}}\right)\right|\left|D^{2} \mathbf{d}\right| d \Omega+\int_{\Omega}\left|\nabla\left(\partial_{t} \mathbf{u}\right)\right|\left|\nabla\left(\partial_{t} \widetilde{\mathbf{d}}\right)\right||\nabla \mathbf{d}| d \Omega=A+B,
$$

then, bounding $A$ as in $J_{4}$ (for the $(H 4)$ condition):

$$
A \leq \bar{\varepsilon}\left\|\partial_{t} \mathbf{u}\right\|_{H^{1}(\Omega)}^{2}+C\left\|\partial_{t} \widetilde{\mathbf{d}}\right\|_{H^{2}(\Omega)}^{2}+C\left\|D^{2} \mathbf{d}\right\|_{L^{q}(\Omega)}^{\frac{2 q}{2 q-3}}\left(\left\|\partial_{t} \mathbf{u}\right\|_{L^{2}(\Omega)}^{2}+\left\|\partial_{t} \widetilde{\mathbf{d}}\right\|_{H^{1}(\Omega)}^{2}\right)
$$

In order to bound $B$, by simplicity we do not use $(H 4)$, but we impose additional regularity on $\widetilde{\mathbf{d}}$, as follows

$$
\begin{aligned}
B & \leq\left\|\nabla\left(\partial_{t} \mathbf{u}\right)\right\|_{L^{2}(\Omega)}\left\|\nabla\left(\partial_{t} \widetilde{\mathbf{d}}\right)\right\|_{L^{3}(\Omega)}\|\nabla \mathbf{d}\|_{L^{6}(\Omega)} \\
& \leq \bar{\varepsilon}\left\|\partial_{t} \mathbf{u}\right\|_{H^{1}(\Omega)}^{2}+C_{\bar{\varepsilon}}\|\mathbf{d}\|_{H^{1}(\Omega)}^{2}\left\|\partial_{t} \widetilde{\mathbf{d}}\right\|_{W^{1,3}(\Omega)}^{2}
\end{aligned}
$$


hence $\partial_{t} \widetilde{\mathbf{d}} \in L^{2}\left(0, T ; \mathbf{W}^{1,3}(\Omega)\right)$ will be imposed.

Integrating by parts the term $\widetilde{J}_{7}$,

$$
\begin{aligned}
\widetilde{J}_{7} & =\int_{\Omega}(\mathbf{u} \cdot \nabla)\left(\partial_{t} \widehat{\mathbf{d}}\right) \cdot \Delta\left(\partial_{t} \widehat{\mathbf{d}}\right) d \Omega+\int_{\Omega}(\mathbf{u} \cdot \nabla)\left(\partial_{t} \widetilde{\mathbf{d}}\right) \cdot \Delta\left(\partial_{t} \widehat{\mathbf{d}}\right) d \Omega \\
& =-\int_{\Omega}(\nabla \mathbf{u} \cdot \nabla)\left(\partial_{t} \widehat{\mathbf{d}}\right) \cdot \nabla\left(\partial_{t} \widehat{\mathbf{d}}\right) d \Omega+\int_{\Omega}(\mathbf{u} \cdot \nabla)\left(\partial_{t} \widetilde{\mathbf{d}}\right) \cdot \Delta\left(\partial_{t} \widehat{\mathbf{d}}\right) d \Omega
\end{aligned}
$$

Observe that the first term of $\widetilde{J}_{7}$ is similar to $J_{4}$, replacing $\mathbf{d}$ by $\widehat{\mathbf{d}}$, hence

$$
\int_{\Omega}|\nabla \mathbf{u}|\left|\nabla\left(\partial_{t} \widehat{\mathbf{d}}\right)\right|^{2} d \Omega \leq \bar{\varepsilon}\left\|\partial_{t} \widehat{\mathbf{d}}\right\|_{H^{2}(\Omega)}^{2}+C_{\bar{\varepsilon}}\|\nabla \mathbf{u}\|_{L^{q}(\Omega)}^{\frac{2 q}{2 q-3}}\left\|\partial_{t} \widehat{\mathbf{d}}\right\|_{H^{1}(\Omega)}^{2}
$$

The second term can be estimated differently depending on the range of $q$ :

- If $\frac{3}{2} \leq q<3$, then:

$$
\begin{gathered}
\int_{\Omega}(\mathbf{u} \cdot \nabla)\left(\partial_{t} \widetilde{\mathbf{d}}\right) \cdot \Delta\left(\partial_{t} \widehat{\mathbf{d}}\right) d \Omega \leq\|\mathbf{u}\|_{L^{3-q}(\Omega)}\left\|\nabla\left(\partial_{t} \widetilde{\mathbf{d}}\right)\right\|_{L^{\frac{6 q}{5 q-6}(\Omega)}}\left\|\Delta\left(\partial_{t} \widehat{\mathbf{d}}\right)\right\|_{L^{2}(\Omega)} \\
\leq C\|\nabla \mathbf{u}\|_{L^{q}(\Omega)}\left\|\nabla\left(\partial_{t} \widetilde{\mathbf{d}}\right)\right\|_{L^{2}(\Omega)}^{\alpha}\left\|\nabla\left(\partial_{t} \widetilde{\mathbf{d}}\right)\right\|_{H^{1}(\Omega)}^{1-\alpha}\left\|\partial_{t} \widehat{\mathbf{d}}\right\|_{H^{2}(\Omega)} \\
\leq \bar{\varepsilon}\left\|\partial_{t} \widehat{\mathbf{d}}\right\|_{H^{2}(\Omega)}^{2}+\bar{\varepsilon}\left\{\left\|\partial_{t} \widetilde{\mathbf{d}}\right\|_{H^{2}(\Omega)}^{2}+\|\nabla \mathbf{u}\|_{L^{q}(\Omega)}^{\frac{2}{\alpha}}\left\|\nabla\left(\partial_{t} \widetilde{\mathbf{d}}\right)\right\|_{L^{2}(\Omega)}^{2}\right\}
\end{gathered}
$$

where $\frac{2}{\alpha}=\frac{2 q}{2 q-3}$.

- If $q \geq 3$, only supposing that $\nabla \mathbf{u} \in L^{\frac{2 q}{2 q-3}}\left(0, T ; \mathbf{L}^{q}(\Omega)\right)$, we cannot bound this term. By simplicity, we will impose some additional regularity for $\partial_{t} \widetilde{\mathbf{d}}$ in order to get this aim.

$$
\begin{aligned}
\int_{\Omega}(\mathbf{u} \cdot \nabla) \nabla\left(\partial_{t} \widetilde{\mathbf{d}}\right) \cdot \nabla\left(\partial_{t} \widehat{\mathbf{d}}\right) d \Omega & \leq\|\mathbf{u}\|_{L^{6}(\Omega)}\left\|D^{2}\left(\partial_{t} \widetilde{\mathbf{d}}\right)\right\|_{L^{3 / 2}(\Omega)}\left\|\nabla\left(\partial_{t} \widehat{\mathbf{d}}\right)\right\|_{L^{6}(\Omega)} \\
& \leq \bar{\varepsilon}\left\|\partial_{t} \widehat{\mathbf{d}}\right\|_{H^{2}(\Omega)}^{2}+C_{\bar{\varepsilon}}\|\mathbf{u}\|_{H^{1}(\Omega)}^{2}\left\|\partial_{t} \widetilde{\mathbf{d}}\right\|_{W^{2,3 / 2}(\Omega)}^{2}
\end{aligned}
$$

(hence $\partial_{t} \widetilde{\mathbf{d}} \in L^{\infty}\left(0, T ; \mathbf{W}^{2,3 / 2}(\Omega)\right)$ will be imposed). 
And therefore, the inequality obtained in the nonhomogeneous Dirichlet boundary case is:

$$
\begin{aligned}
& \frac{d}{d t}\left(\lambda^{-1}\left\|\partial_{t} \mathbf{u}\right\|_{L^{2}(\Omega)}^{2}+\left\|\partial_{t} \widehat{\mathbf{d}}\right\|_{H^{1}(\Omega)}^{2}\right)+\frac{\nu}{\lambda}\left\|\partial_{t} \mathbf{u}\right\|_{H^{1}(\Omega)}^{2}+\left\|\partial_{t} \widehat{\mathbf{d}}\right\|_{H^{2}(\Omega)}^{2} \\
& \quad \leq C\left(\|\nabla \mathbf{u}\|_{L^{q}(\Omega)}^{\frac{2 q}{2 q-3}}+\left[\|\nabla \mathbf{d}\|_{L^{p}(\Omega)}^{\frac{2 p}{p-3}} \text { or }\|\Delta \mathbf{d}\|_{L^{q}(\Omega)}^{\frac{2 q}{2-3}}+\|\mathbf{d}\|_{L^{2}(\Omega}^{\frac{2 q}{2 q-3}}\right]\right) \\
& \quad \times\left(\left\|\partial_{t} \mathbf{u}\right\|_{L^{2}(\Omega)}^{2}+\left\|\partial_{t} \widehat{\mathbf{d}}\right\|_{H^{1}(\Omega)}^{2}+\left\|\partial_{t} \widetilde{\mathbf{d}}\right\|_{H^{1}(\Omega)}^{2}\right) \\
& \quad+C\left\{\|\mathbf{u}\|_{H^{1}(\Omega)}^{2}\left\|\partial_{t} \widetilde{\mathbf{d}}\right\|_{W^{2,3 / 2}(\Omega)}^{2}+\left\|\partial_{t} \widehat{\mathbf{d}}\right\|_{L^{2}(\Omega)}^{2}+\left\|\partial_{t} \widetilde{\mathbf{d}}\right\|_{L^{2}(\Omega)}^{2}\right. \\
& \left.\quad+\mu\|\mathbf{d}\|_{H^{1}(\Omega)}^{2}\left\|\partial_{t} \widetilde{\mathbf{d}}\right\|_{W^{1,3}(\Omega)}^{2}\right\},
\end{aligned}
$$

where $\mu=0$ if (H3) is supposed and $\mu=1$ if $(H 4)$.

\section{Regularity for the periodic boundary condition case (proof of Theorem 1.3 with (H1))}

Attending to the development of the previous section, if we only impose the regularity hypothesis $(H 1)$ on $\nabla \mathbf{u}$ but not hypothesis $(H 3)$ for $\nabla \mathbf{d}$ nor hypothesis $(H 4)$ for $\Delta \mathbf{d}$, for the exponent $q \in(3 / 2,2) \cup(3,+\infty)$ we cannot assure that $\mathbf{d} \in L^{\infty}\left(0, T ; \mathbf{H}^{2}(\Omega)\right) \cap$ $L^{2}\left(0, T ; \mathbf{H}^{3}(\Omega)\right)$, nor that $\mathbf{u} \in L^{\infty}\left(0, T ; \mathbf{H}^{1}(\Omega)\right) \cap L^{2}\left(0, T ; \mathbf{H}^{2}(\Omega)\right)$. In a attempt to improve the regularity for $\mathbf{d}$ (without imposing additional regularity hypothesis for $\mathbf{d}$ ) and in the case of periodic boundary conditions for $\mathbf{d}$, we will study a certain non-hilbertian regularity. To this aim, we take $-\nabla \cdot(|\nabla \mathbf{d}| \nabla \mathbf{d})$ as test function in the system for $\mathbf{d}$, where separating term by term and integrating by parts (the boundary integral vanish thanks to the periodic boundary conditions on $\mathbf{d}$ ), we obtain:

$$
-\int_{\Omega} \partial_{t} \mathbf{d} \cdot[\nabla \cdot(|\nabla \mathbf{d}| \nabla \mathbf{d})] d \Omega=\int_{\Omega} \partial_{t}(\nabla \mathbf{d}) \cdot \nabla \mathbf{d}|\nabla \mathbf{d}| d \Omega=\frac{1}{3} \frac{d}{d t}\|\nabla \mathbf{d}\|_{L^{3}(\Omega)}^{3}
$$




$$
\begin{aligned}
\int_{\Omega} \Delta \mathbf{d} \cdot[\nabla \cdot(|\nabla \mathbf{d}| \nabla \mathbf{d})] d \Omega & =-\int_{\Omega} \nabla(\Delta \mathbf{d}) \nabla \mathbf{d}|\nabla \mathbf{d}| d \Omega=\int_{\Omega} D^{2} \mathbf{d} \nabla(\nabla \mathbf{d}|\nabla \mathbf{d}|) d \Omega \\
& =\int_{\Omega}\left|D^{2} \mathbf{d}\right|^{2}|\nabla \mathbf{d}| d \Omega+\left(\frac{2}{3}\right)^{2} \int_{\Omega}\left|\nabla\left(|\nabla \mathbf{d}|^{3 / 2}\right)\right|^{2} d \Omega \\
\int_{\Omega}(\mathbf{u} \cdot \nabla) \mathbf{d} \cdot \nabla \cdot(|\nabla \mathbf{d}| \nabla \mathbf{d}) d \Omega & =-\int_{\Omega} \nabla \mathbf{u} \cdot \nabla \mathbf{d}|\nabla \mathbf{d}| \nabla \mathbf{d} d \Omega-\int_{\Omega}(\mathbf{u} \cdot \nabla) \frac{|\nabla \mathbf{d}|^{2}}{2}|\nabla \mathbf{d}| d \Omega \\
& \leq\left. C \int_{\Omega}|\nabla \mathbf{u}| \nabla \mathbf{d}\right|^{3} d \Omega-\frac{1}{3} \int_{\Omega}(\mathbf{u} \cdot \nabla)\left(|\nabla \mathbf{d}|^{3}\right) d \Omega \\
& \leq\left. C \int_{\Omega}|\nabla \mathbf{u}| \nabla \mathbf{d}\right|^{3} d \Omega
\end{aligned}
$$

We arrive to the inequality:

$$
\begin{aligned}
\frac{1}{3} \frac{d}{d t}\|\nabla \mathbf{d}\|_{L^{3}(\Omega)}^{3} & +\gamma \int_{\Omega}\left|D^{2} \mathbf{d}\right|^{2}|\nabla \mathbf{d}| d \Omega+\gamma\left(\frac{2}{3}\right)^{2}\left\|\nabla\left(|\nabla \mathbf{d}|^{3 / 2}\right)\right\|_{L^{2}(\Omega)}^{2} d \Omega \\
& \leq C \int_{\Omega}|\nabla \mathbf{u}||\nabla \mathbf{d}|^{3} d \Omega-\gamma \int_{\Omega} \mathbf{f}_{\delta}(\mathbf{d}) \nabla \cdot(|\nabla \mathbf{d}| \nabla \mathbf{d}) d \Omega
\end{aligned}
$$

The bound for the first term on the right hand side is:

$$
\begin{aligned}
\int_{\Omega}|\nabla \mathbf{u}||\nabla \mathbf{d}|^{3} d \Omega & \leq\|\nabla \mathbf{u}\|_{L^{q}(\Omega)}\|\nabla \mathbf{d}\|_{L^{\frac{3 q}{q-1}}(\Omega)} \\
& \leq\|\nabla \mathbf{u}\|_{L^{q}(\Omega)}\|\nabla \mathbf{d}\|_{L^{3}(\Omega)}^{3 \alpha}\|\nabla \mathbf{d}\|_{L^{9}(\Omega)}^{3(1-\alpha)} \\
& \leq\|\nabla \mathbf{u}\|_{L^{q}(\Omega)}\|\nabla \mathbf{d}\|_{L^{3}(\Omega)}^{3 \alpha}\left\|\nabla\left(|\nabla \mathbf{d}|^{3 / 2}\right)\right\|_{L^{2}(\Omega)}^{2(1-\alpha)} \\
& \leq \bar{\varepsilon}\left\|\nabla\left(|\nabla \mathbf{d}|^{3 / 2}\right)\right\|_{L^{2}(\Omega)}^{2}+C_{\bar{\varepsilon}}\|\nabla \mathbf{u}\|_{L^{q}(\Omega)}^{1 / \alpha}\|\nabla \mathbf{d}\|_{L^{3}(\Omega)}^{3}
\end{aligned}
$$

with $\frac{1}{\alpha}=\frac{2 q}{2 q-3}$. Here, we have used the inequality ([4]):

$$
\|\nabla \mathbf{d}\|_{L^{9}(\Omega)}^{9} \leq\left\||\nabla \mathbf{d}|^{3 / 2}\right\|_{L^{6}(\Omega)}^{6} \leq C\left\|\nabla\left(|\nabla \mathbf{d}|^{3 / 2}\right)\right\|_{L^{2}(\Omega)}^{6}
$$

The bound we get for the term $\mathbf{f}_{\delta}(\mathbf{d})$ is:

$$
\begin{aligned}
\int_{\Omega} \mathbf{f}_{\delta}(\mathbf{d}) \nabla \cdot(|\nabla \mathbf{d}| \nabla \mathbf{d}) d \Omega & \leq C \int_{\Omega}|\mathbf{d}|^{3}|\nabla \mathbf{d}|\left|D^{2} \mathbf{d}\right| d \Omega \leq C \int_{\Omega}|\nabla \mathbf{d}|\left|D^{2} \mathbf{d}\right| d \Omega \\
& \leq \bar{\varepsilon} \int_{\Omega}|\nabla \mathbf{d}|\left|D^{2} \mathbf{d}\right|^{2} d \Omega+C_{\bar{\varepsilon}} \int_{\Omega}|\nabla \mathbf{d}| d \Omega
\end{aligned}
$$


Therefore, only imposing the additional regularity (H1) for $\nabla \mathbf{u}$, we can conclude that:

$$
\left\{\begin{aligned}
\nabla \mathbf{d} & \in L^{\infty}\left(0, T ; \mathbf{L}^{3}(\Omega)\right), \\
\nabla\left(|\nabla \mathbf{d}|^{3 / 2}\right) & \in L^{2}\left(0, T ; \mathbf{L}^{2}(\Omega)\right), \\
\int_{\Omega}|\nabla \mathbf{d}||\Delta \mathbf{d}|^{2} & \in L^{1}(0, T)
\end{aligned}\right.
$$

Moreover, from $\nabla\left(|\nabla \mathbf{d}|^{3 / 2}\right) \in L^{2}\left(0, T ; \mathbf{L}^{2}(\Omega)\right)$ and the inequality (32), one can be deduced that $\nabla \mathbf{d} \in L^{3}\left(0, T ; \mathbf{L}^{9}(\Omega)\right)$.

In consequence, in the periodic boundary condition case, the additional regularity (H3) which was necessary in order to obtain the strong regularity for $(L C)$, is verified for the exponent $p=9$. Therefore, the only additional hypothesis to impose is $(H 1)$, that is, $\nabla \mathbf{u} \in L^{\frac{2 q}{2 q-3}}\left(0, T ; \mathbf{L}^{q}(\Omega)\right)$ for any $q>3 / 2$.

\section{$5 \quad$ Uniqueness (proof of Theorem 1.4 with (H1))}

We consider the systems verified by $\overline{\mathbf{u}}=\mathbf{u}_{1}-\mathbf{u}_{2}, \overline{\mathbf{d}}=\mathbf{d}_{1}-\mathbf{d}_{2}$ and $\bar{\pi}=\pi_{1}-\pi_{2}$, where $\left(\mathbf{u}_{1}, \mathbf{d}_{1}, \pi_{1}\right)$ and $\left(\mathbf{u}_{2}, \mathbf{d}_{2}, \pi_{2}\right)$ are two solutions of the Nematic Liquid Crystal system $(L C)$, respectively. Then:

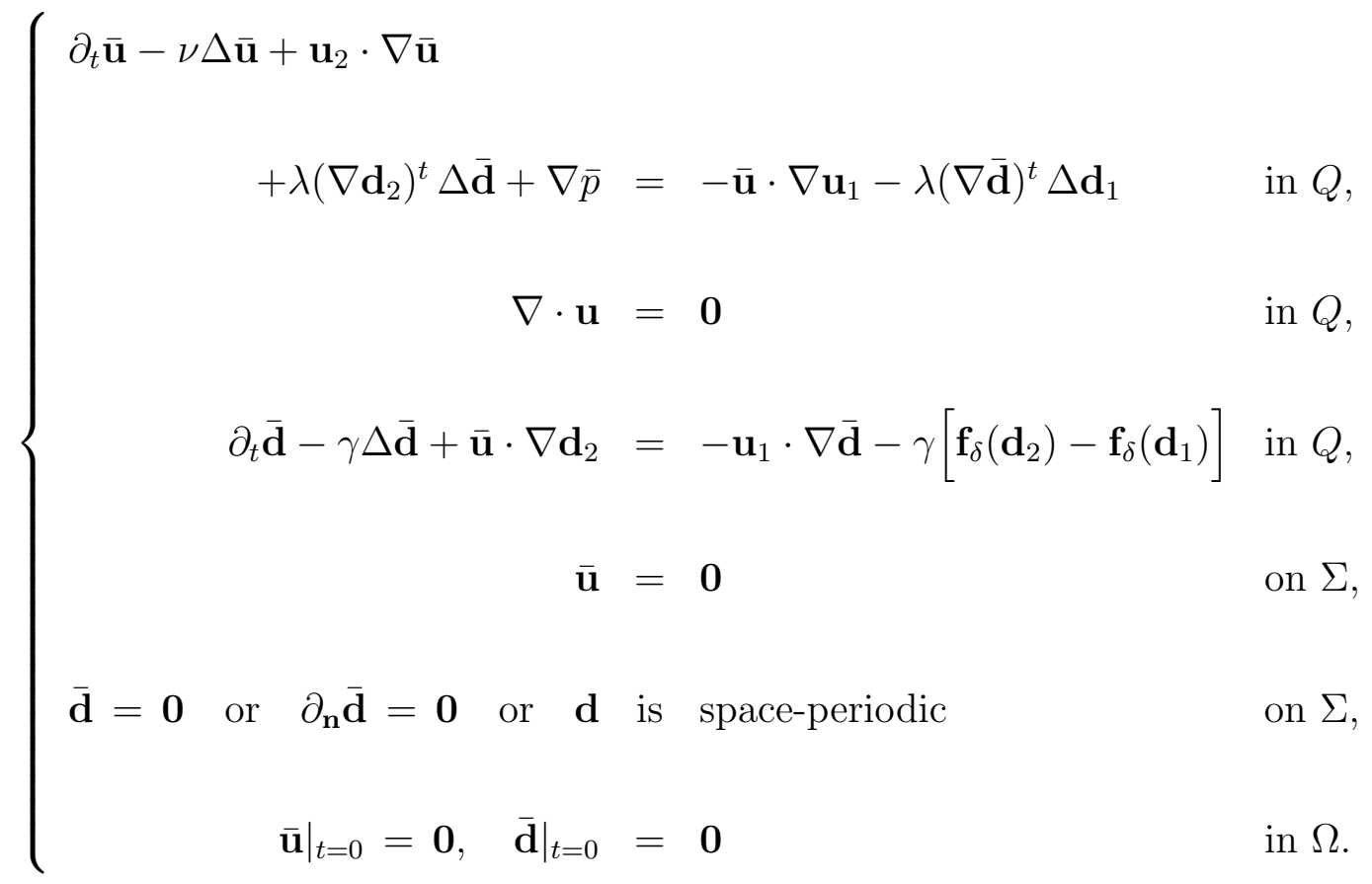


Arguing in a formal way, we take $\overline{\mathbf{d}}$ as test functions in the system for $\overline{\mathbf{d}}$ and use the equality:

$$
\mathbf{f}_{\delta}\left(\mathbf{d}_{2}\right)-\mathbf{f}_{\delta}\left(\mathbf{d}_{1}\right)=-\frac{1}{\delta^{2}}\left[\left(\left|\mathbf{d}_{1}\right|^{2}-1\right) \overline{\mathbf{d}}+\left(\left(\mathbf{d}_{1}+\mathbf{d}_{2}\right) \cdot \overline{\mathbf{d}}\right) \mathbf{d}_{2}\right]
$$

obtaining:

$$
\begin{aligned}
\frac{1}{2} \frac{d}{d t}\|\overline{\mathbf{d}}\|_{L^{2}(\Omega)}^{2} & +\gamma\|\nabla \overline{\mathbf{d}}\|_{L^{2}(\Omega)}^{2}=-\int_{\Omega}(\overline{\mathbf{u}} \cdot \nabla) \mathbf{d}_{2} \cdot \overline{\mathbf{d}} d \Omega \\
& -\gamma \int_{\Omega}\left(\left|\mathbf{d}_{1}\right|^{2}-1\right)|\overline{\mathbf{d}}|^{2} d \Omega-\gamma \int_{\Omega}\left[\left(\mathbf{d}_{1}+\mathbf{d}_{2}\right) \cdot \overline{\mathbf{d}}\right]\left(\mathbf{d}_{2} \cdot \overline{\mathbf{d}}\right) d \Omega
\end{aligned}
$$

which can be rewritten as (using that $\left|\mathbf{d}_{1}\right|$ and $\left|\mathbf{d}_{2}\right| \leq 1$ ):

$$
\begin{aligned}
\frac{1}{2} \frac{d}{d t}\|\overline{\mathbf{d}}\|_{L^{2}(\Omega)}^{2} & +\gamma\|\nabla \overline{\mathbf{d}}\|_{L^{2}(\Omega)}^{2} \leq \int_{\Omega}|\overline{\mathbf{u}}||\nabla \overline{\mathbf{d}}|\left|\mathbf{d}_{2}\right| d \Omega+C\|\overline{\mathbf{d}}\|_{L^{2}(\Omega)}^{2} \\
& \leq \bar{\varepsilon}\|\nabla \overline{\mathbf{d}}\|_{L^{2}(\Omega)}^{2}+C_{\bar{\varepsilon}}\|\overline{\mathbf{u}}\|_{L^{2}(\Omega)}^{2}+C\|\overline{\mathbf{d}}\|_{L^{2}(\Omega)}^{2}
\end{aligned}
$$

Thus, we obtain:

$$
\frac{d}{d t}\|\overline{\mathbf{d}}\|_{L^{2}(\Omega)}^{2}+\gamma\|\nabla \overline{\mathbf{d}}\|_{L^{2}(\Omega)}^{2} \leq C\left(\gamma\|\overline{\mathbf{d}}\|_{L^{2}(\Omega)}^{2}+\|\overline{\mathbf{u}}\|_{L^{2}(\Omega)}^{2}\right)
$$

Now, we add the estimates obtained taking $\lambda^{-1} \overline{\mathbf{u}}$ as test functions in the system for $\overline{\mathbf{u}}$, and $-\Delta \overline{\mathbf{d}}$ as test functions in the system for $\overline{\mathbf{d}}$. Observe that the terms $\int_{\Omega} \overline{\mathbf{u}} \cdot \nabla \mathbf{d}_{2} \cdot \Delta \overline{\mathbf{d}}$ cancel. Moreover, adding (35) to the resulting estimate, we obtain:

$$
\begin{aligned}
& \frac{d}{d t}\left(\lambda^{-1}\|\overline{\mathbf{u}}\|_{L^{2}(\Omega)}^{2}+\|\overline{\mathbf{d}}\|_{H^{1}(\Omega)}^{2}\right)+\frac{\nu}{\lambda}\|\overline{\mathbf{u}}\|_{H^{1}(\Omega)}^{2}+\gamma\|\nabla \overline{\mathbf{d}}\|_{H^{1}(\Omega)}^{2} \leq \\
& -\lambda^{-1} \int_{\Omega} \overline{\mathbf{u}} \cdot \nabla \mathbf{u}_{1} \overline{\mathbf{u}} d \Omega-\int_{\Omega} \overline{\mathbf{u}} \cdot \nabla \overline{\mathbf{d}} \Delta \mathbf{d}_{1} d \Omega+\int_{\Omega} \mathbf{u}_{1} \cdot \nabla \overline{\mathbf{d}} \Delta \overline{\mathbf{d}} d \Omega \\
& +\gamma \int_{\Omega}\left(\mathbf{f}_{\delta}\left(\mathbf{d}_{2}\right)-\mathbf{f}_{\delta}\left(\mathbf{d}_{1}\right)\right) \cdot \Delta \overline{\mathbf{d}} d \Omega+C\left(\|\overline{\mathbf{u}}\|_{L^{2}(\Omega)}^{2}+\|\overline{\mathbf{d}}\|_{L^{2}(\Omega)}^{2}\right)
\end{aligned}
$$

The terms on the right hand side of (36) can be bound as follows:

$$
\begin{aligned}
-\int_{\Omega} \overline{\mathbf{u}} \cdot \nabla \mathbf{u}_{1} \overline{\mathbf{u}} d \Omega & \leq \int_{\Omega}|\overline{\mathbf{u}}|^{2}\left|\nabla \mathbf{u}_{1}\right| d \Omega \leq\|\overline{\mathbf{u}}\|_{L^{\frac{2 q}{q-1}}(\Omega)}^{2}\left\|\nabla \mathbf{u}_{1}\right\|_{L^{q}(\Omega)} \\
& \leq\|\overline{\mathbf{u}}\|_{L^{2}(\Omega)}^{\frac{2 q-3}{q}}\|\nabla \overline{\mathbf{u}}\|_{L^{2}(\Omega)}^{\frac{3}{q}}\left\|\nabla \mathbf{u}_{1}\right\|_{L^{q}(\Omega)} \\
& \leq \bar{\varepsilon}\|\overline{\mathbf{u}}\|_{H^{1}(\Omega)}^{2}+C_{\bar{\varepsilon}}\left\|\nabla \mathbf{u}_{1}\right\|_{L^{q}(\Omega)}^{\frac{2 q}{2 q-3}}\|\overline{\mathbf{u}}\|_{L^{2}(\Omega)}^{2}
\end{aligned}
$$


In order to bound the third term, we rewrite it in the form:

$$
\begin{gathered}
\int_{\Omega} \mathbf{u}_{1} \cdot \nabla \overline{\mathbf{d}} \Delta \overline{\mathbf{d}} d \Omega \\
=-\int_{\Omega} \nabla \mathbf{u}_{1} \cdot \nabla \overline{\mathbf{d}} \nabla \overline{\mathbf{d}} d \Omega \leq \int_{\Omega}\left|\nabla \mathbf{u}_{1}\right||\nabla \overline{\mathbf{d}}|^{2} d \Omega \\
\leq\left\|\nabla \mathbf{u}_{1}\right\|_{L^{q}(\Omega)}\|\nabla \overline{\mathbf{d}}\|_{L^{\frac{2 q}{q-1}}(\Omega)}^{2} \\
\leq \bar{\varepsilon}\|\nabla \overline{\mathbf{d}}\|_{H^{1}(\Omega)}^{2}+C_{\bar{\varepsilon}}\|\nabla \mathbf{u}\|_{L^{q}(\Omega)}^{\frac{2 q}{2-3}}\|\nabla \overline{\mathbf{d}}\|_{L^{2}(\Omega)}^{2} \\
\int_{\Omega}\left(\mathbf{f}_{\delta}\left(\mathbf{d}_{2}\right)-\mathbf{f}_{\delta}\left(\mathbf{d}_{1}\right)\right)|\Delta \overline{\mathbf{d}}| d \Omega \leq C \int_{\Omega}|\overline{\mathbf{d}}||\Delta \overline{\mathbf{d}}| d \Omega \leq \bar{\varepsilon}\|\Delta \overline{\mathbf{d}}\|_{L^{2}(\Omega)}^{2}+C_{\bar{\varepsilon}}\|\overline{\mathbf{d}}\|_{L^{2}(\Omega)}^{2}
\end{gathered}
$$

In order to bound the second term, we can proceed in two different ways (as in previous arguments):

1. If we integrate directly, we obtain:

$$
\begin{aligned}
-\int_{\Omega} \overline{\mathbf{u}} \cdot \nabla \overline{\mathbf{d}} \Delta \mathbf{d}_{1} d \Omega & \leq\|\overline{\mathbf{u}}\|_{L^{\frac{2 q}{q-1}(\Omega)}}\|\nabla \overline{\mathbf{d}}\|_{L^{\frac{2 q}{q-1}(\Omega)}}\left\|\Delta \mathbf{d}_{1}\right\|_{L^{q}(\Omega)} \\
& \leq\|\overline{\mathbf{u}}\|_{L^{2}(\Omega)}^{\alpha}\|\nabla \overline{\mathbf{u}}\|_{L^{2}(\Omega)}^{1-\alpha}\|\nabla \overline{\mathbf{d}}\|_{L^{2}(\Omega)}^{\alpha}\|\nabla \overline{\mathbf{d}}\|_{H^{1}(\Omega)}^{1-\alpha}\left\|\Delta \mathbf{d}_{1}\right\|_{L^{q}(\Omega)} \\
& \leq \bar{\varepsilon}\left(\|\nabla \overline{\mathbf{u}}\|_{L^{2}(\Omega)}^{2}+\|\nabla \overline{\mathbf{d}}\|_{H^{1}(\Omega)}^{2}\right) \\
& +C_{\bar{\varepsilon}}\left\|\Delta \mathbf{d}_{1}\right\|_{L^{q}(\Omega)}^{\frac{1}{\alpha}}\left(\|\overline{\mathbf{u}}\|_{L^{2}(\Omega)}^{2}+\|\nabla \overline{\mathbf{d}}\|_{L^{2}(\Omega)}^{2}\right)
\end{aligned}
$$

In this case, starting from (36), we obtain the following inequality:

$$
\begin{aligned}
& \frac{d}{d t}\left(\lambda^{-1}\|\overline{\mathbf{u}}\|_{L^{2}(\Omega)}^{2}+\|\overline{\mathbf{d}}\|_{H^{1}(\Omega)}^{2}\right)+\frac{\nu}{\lambda}\|\overline{\mathbf{u}}\|_{H^{1}(\Omega)}^{2}+\gamma\|\nabla \overline{\mathbf{d}}\|_{H^{1}(\Omega)}^{2} \\
& \leq C\left(\left\|\nabla \mathbf{u}_{1}\right\|_{L^{q_{1}(\Omega)}}^{\frac{2 q_{1}}{2 q_{1}-3}}+\left\|\Delta \mathbf{d}_{1}\right\|_{L^{q_{2}(\Omega)}}^{\frac{2 q_{2}}{2 q_{2}}}+1\right)\left(\|\overline{\mathbf{u}}\|_{L^{2}(\Omega)}^{2}+\|\overline{\mathbf{d}}\|_{H^{1}(\Omega)}^{2}\right)
\end{aligned}
$$

that thanks to the Gronwall's Lemma allows us to conclude that $\overline{\mathbf{u}}=\mathbf{0}$ and $\overline{\mathbf{d}}=\mathbf{0}$. Then, assuming that $\mathbf{u}_{1}$ verifies the additional regularity $(H 1)$ and $\mathbf{d}_{1}$ verifes $(H 4)$, the uniqueness is attempt. 
2. If we integrate by parts, the third term can be written as:

$$
\begin{aligned}
& -\int_{\Omega} \overline{\mathbf{u}} \cdot \nabla \overline{\mathbf{d}} \Delta \mathbf{d}_{1} d \Omega=\int_{\Omega} \nabla \overline{\mathbf{u}} \cdot \nabla \mathbf{d} \cdot \nabla \overline{\mathbf{d}}_{1} d \Omega+\int_{\Omega}(\overline{\mathbf{u}} \cdot \nabla) \nabla \overline{\mathbf{d}} \cdot \nabla \mathbf{d}_{1} d \Omega \\
\leq & \int_{\Omega}\left(|\nabla \overline{\mathbf{u}} \| \nabla \overline{\mathbf{d}}|+|\overline{\mathbf{u}}|\left|D^{2} \overline{\mathbf{d}}\right|\right)\left|\nabla \mathbf{d}_{1}\right| d \Omega \\
\leq & \left(\|\nabla \overline{\mathbf{u}}\|_{L^{2}(\Omega)}\|\nabla \overline{\mathbf{d}}\|_{L^{\frac{2 p}{p-2}}(\Omega)}+\|\overline{\mathbf{u}}\|_{L^{\frac{2 p}{p-2}}(\Omega)}\left\|D^{2} \overline{\mathbf{d}}\right\|_{L^{2}(\Omega)}\right)\left\|\nabla \mathbf{d}_{1}\right\|_{L^{p}(\Omega)} \\
\leq & \bar{\varepsilon}\left(\|\nabla \overline{\mathbf{u}}\|_{L^{2}(\Omega)}^{2}+\|\Delta \overline{\mathbf{d}}\|_{L^{2}(\Omega)}^{2}\right)+C_{\bar{\varepsilon}}\left\|\nabla \mathbf{d}_{1}\right\|_{L^{p}(\Omega)}^{\frac{2 p}{p-3}}\left(\|\overline{\mathbf{u}}\|_{L^{2}(\Omega)}^{2}+\|\nabla \overline{\mathbf{d}}\|_{L^{2}(\Omega)}^{2}\right)
\end{aligned}
$$

In this case, the inequality (37) is obtained, replacing $\left\|\Delta \mathbf{d}_{1}\right\|_{L^{p}(\Omega)}^{\frac{2 p}{2 p-3}}$ by $\left\|\nabla \mathbf{d}_{1}\right\|_{L^{p}(\Omega)}^{\frac{2 p}{p-3}}$. In consequence, the Gronwall's Lemma conclude the uniqueness, supposing the additional regularity $(H 1)$ and $(H 3)$.

\section{Variants for the Serrin's class (H2).}

Until now, we have supposed that $\mathbf{u}$ has regularity of Berselli type $(H 1)$, see[3]. Now, our aim is to study the influence of the regularity hypothesis $(H 2)$ on $\mathbf{u}$ in order to obtain strong regularity (global in time) and uniqueness of solution.

By simplicity, we focus on the Neumann case, remarking the Dirichlet case at the end.

\subsection{Outline of the proof of Theorem 1.2}

Following the same procedure then in Section 3, we obtain an estimate similar to (24) but where $J_{2}$ and $J_{3}$ are replaced by:

$$
\int_{\Omega}(\mathbf{u} \cdot \nabla) \partial_{t} \mathbf{d} \cdot \Delta\left(\partial_{t} \mathbf{d}\right) d \Omega-\frac{1}{\lambda} \int_{\Omega}\left(\partial_{t} \mathbf{u} \cdot \nabla\right) \mathbf{u} \cdot \partial_{t} \mathbf{u} d \Omega:=\widetilde{J}_{2}+\widetilde{J}_{3}
$$

which can be bound as follows:

$$
\begin{aligned}
\left|\widetilde{J}_{2}\right| & \leq\|\mathbf{u}\|_{L^{p}(\Omega)}\left\|\nabla\left(\partial_{t} \mathbf{d}\right)\right\|_{L^{\frac{2 p}{p-2}}(\Omega)}\left\|\Delta\left(\partial_{t} \mathbf{d}\right)\right\|_{L^{2}(\Omega)} \\
& \leq\|\mathbf{u}\|_{L^{p}(\Omega)}\left\|\nabla\left(\partial_{t} \mathbf{d}\right)\right\|_{L^{2}(\Omega)}^{\beta}\left\|\nabla\left(\partial_{t} \mathbf{d}\right)\right\|_{H^{1}(\Omega)}^{2-\beta} \\
& \leq \bar{\varepsilon}\left\|\nabla\left(\partial_{t} \mathbf{d}\right)\right\|_{H^{1}(\Omega)}^{2}+C_{\bar{\varepsilon}}\|\mathbf{u}\|_{L^{p}(\Omega)}^{\frac{2}{\beta}}\left\|\nabla\left(\partial_{t} \mathbf{d}\right)\right\|_{L^{2}(\Omega)}^{2}
\end{aligned}
$$


where $\frac{2}{\beta}=\frac{2 p}{p-3}$. The $\widetilde{J}_{3}$-term can be rewritten as:

$$
\begin{aligned}
\widetilde{J}_{3} & =\frac{1}{\lambda} \int_{\Omega}\left(\partial_{t} \mathbf{u} \cdot \nabla\right) \partial_{t} \mathbf{u} \cdot \mathbf{u} d \Omega \\
& \leq \frac{1}{\lambda}\|\mathbf{u}\|_{L^{p}(\Omega)}\left\|\partial_{t} \mathbf{u}\right\|_{L^{\frac{2 p}{p-2}(\Omega)}}\left\|\nabla\left(\partial_{t} \mathbf{u}\right)\right\|_{L^{2}(\Omega)} \\
& \leq C\|\mathbf{u}\|_{L^{p}(\Omega)}\left\|\partial_{t} \mathbf{u}\right\|_{L^{2}(\Omega)}^{\beta}\left\|\nabla\left(\partial_{t} \mathbf{u}\right)\right\|_{L^{2}(\Omega)}^{2-\beta} \\
& \leq \bar{\varepsilon}\left\|\nabla\left(\partial_{t} \mathbf{u}\right)\right\|_{L^{2}(\Omega)}^{2}+C_{\bar{\varepsilon}}\|\mathbf{u}\|_{L^{p}(\Omega)}^{\frac{2}{\beta}}\left\|\partial_{t} \mathbf{u}\right\|_{L^{2}(\Omega)}^{2}
\end{aligned}
$$

Remark 6.1 In the nonhomogeneous Dirichlet boundary condition case, the only terms that differs from the Neumann case is $\int_{\Omega}(\mathbf{u} \cdot \nabla) \partial_{t} \mathbf{d} \cdot \Delta\left(\partial_{t} \widehat{\mathbf{d}}\right) d \Omega$, but it can be bounded in a similar manner as follows:

$$
\int_{\Omega}(\mathbf{u} \cdot \nabla) \partial_{t} \mathbf{d} \cdot \Delta\left(\partial_{t} \widehat{\mathbf{d}}\right) d \Omega \leq \bar{\varepsilon}\left(\left\|\partial_{t} \widehat{\mathbf{d}}\right\|_{H^{2}(\Omega)}^{2}+\left\|\partial_{t} \widetilde{\mathbf{d}}\right\|_{H^{2}(\Omega)}^{2}\right)+C_{\bar{\varepsilon}}\|\mathbf{u}\|_{L^{p}(\Omega)}^{\frac{2}{\beta}}\left\|\nabla\left(\partial_{t} \mathbf{d}\right)\right\|_{L^{2}(\Omega)}^{2}
$$

\subsection{Outline of the proof of Theorem 1.3}

In this case, the difficulty arises in estimating the nonlinear term, that now is decomposed in a different manner as follows:

$$
\begin{aligned}
\int_{\Omega}(\mathbf{u} \cdot \nabla) \mathbf{d} \cdot[\nabla \cdot(|\nabla \mathbf{d}| \nabla \mathbf{d})] d \Omega & =\int_{\Omega}(\mathbf{u} \cdot \nabla) \mathbf{d} \cdot \Delta \mathbf{d}|\nabla \mathbf{d}| d \Omega \\
& +\int_{\Omega}(\mathbf{u} \cdot \nabla) \mathbf{d} \cdot \nabla \mathbf{d} \frac{\nabla \mathbf{d} \cdot \nabla(\nabla \mathbf{d})}{|\nabla \mathbf{d}|} d \Omega \\
& \leq C \int_{\Omega}|\mathbf{u}||\nabla \mathbf{d}|^{2}\left|D^{2} \mathbf{d}\right| d \Omega \\
& \leq \bar{\varepsilon} \int_{\Omega}|\nabla \mathbf{d}|\left|D^{2} \mathbf{d}\right|^{2} d \Omega+C_{\bar{\varepsilon}} \int_{\Omega}|\mathbf{u}|^{2}|\nabla \mathbf{d}|^{3} d \Omega
\end{aligned}
$$

where

$$
\begin{aligned}
\int_{\Omega}|\mathbf{u}|^{2}|\nabla \mathbf{d}|^{3} d \Omega & \leq\|\mathbf{u}\|_{L^{p}(\Omega)}^{2}\|\nabla \mathbf{d}\|_{L^{\frac{3 p}{p-2}(\Omega)}}^{3} \leq\|\mathbf{u}\|_{L^{p}(\Omega)}^{2}\|\nabla \mathbf{d}\|_{L^{2}(\Omega)}^{3 \beta}\|\nabla \mathbf{d}\|_{L^{9}(\Omega)}^{3(1-\beta)} \\
& \leq\|\mathbf{u}\|_{L^{p}(\Omega)}^{2}\|\nabla \mathbf{d}\|_{L^{3}(\Omega)}^{3 \beta}\left\|\nabla\left(|\nabla \mathbf{d}|^{3 / 2}\right)\right\|_{L^{2}(\Omega)}^{2(1-\beta)} \\
& \leq \bar{\varepsilon}\left\|\nabla\left(|\nabla \mathbf{d}|^{3 / 2}\right)\right\|_{L^{2}(\Omega)}^{2}+C_{\bar{\varepsilon}}\|\mathbf{u}\|_{L^{p}(\Omega)}^{\frac{2}{\beta}}\|\nabla \mathbf{d}\|_{L^{3}(\Omega)}^{3}
\end{aligned}
$$


and $\frac{2}{\beta}=\frac{2 p}{p-3}($ recall that $p>3)$.

\subsection{Outline of the proof of Theorem 1.4 (uniqueness)}

Starting from the expression (36), we bound the terms on the right hand side using the new additional regularity hypothesis $(H 2)$ for $\mathbf{u}$ and $(H 3)$ for $\mathbf{d}$.

$$
\begin{aligned}
& -\int_{\Omega}(\overline{\mathbf{u}} \cdot \nabla) \mathbf{u}_{1} \overline{\mathbf{u}} d \Omega=\int_{\Omega} \overline{\mathbf{u}} \cdot \nabla \overline{\mathbf{u}} \mathbf{u}_{1} d \Omega \\
& \leq\|\overline{\mathbf{u}}\|_{L^{\frac{2 p}{p-2}(\Omega)}}\|\nabla \overline{\mathbf{u}}\|_{L^{2}(\Omega)}\left\|\mathbf{u}_{1}\right\|_{L^{p}(\Omega)} \\
& \leq\|\overline{\mathbf{u}}\|_{L^{2}(\Omega)}^{\beta}\|\nabla \overline{\mathbf{u}}\|_{L^{2}(\Omega)}^{2-\beta}\left\|\mathbf{u}_{1}\right\|_{L^{p}(\Omega)} \\
& \leq \bar{\varepsilon}\|\nabla \overline{\mathbf{u}}\|_{L^{2}(\Omega)}^{2}+C_{\bar{\varepsilon}}\left\|\mathbf{u}_{1}\right\|_{L^{p}(\Omega)}^{\frac{2}{\beta}}\|\overline{\mathbf{u}}\|_{L^{2}(\Omega)}^{2} \\
& \int_{\Omega} \mathbf{u}_{1} \cdot \nabla \overline{\mathbf{d}} \Delta \overline{\mathbf{d}} d \Omega \leq\left\|\mathbf{u}_{1}\right\|_{L^{p}(\Omega)}\|\nabla \overline{\mathbf{d}}\|_{L^{\frac{2 p}{p-2}}(\Omega)}\|\Delta \overline{\mathbf{d}}\|_{L^{2}(\Omega)} \\
& \leq\left\|\mathbf{u}_{1}\right\|_{L^{p}(\Omega)}\|\nabla \overline{\mathbf{d}}\|_{L^{2}(\Omega)}^{\beta}\|\nabla \overline{\mathbf{d}}\|_{H^{1}(\Omega)}^{2-\beta} \\
& \leq \bar{\varepsilon}\|\nabla \overline{\mathbf{d}}\|_{H^{1}(\Omega)}^{2}+C_{\bar{\varepsilon}}\left\|\mathbf{u}_{1}\right\|_{L^{p}(\Omega)}^{\frac{2}{\beta}}\|\nabla \overline{\mathbf{d}}\|_{L^{2}(\Omega)}^{2}
\end{aligned}
$$

where $\frac{2}{\beta}=\frac{2 p}{p-3}$ for $p \geq 3$.

The second term on the right hand side of (36) can be bounded as in Section 5. In consequence, we arrive to $(37)$ replacing $\left\|\nabla \mathbf{u}_{1}\right\|_{L^{p}(\Omega)}^{\frac{2 q}{2 q-3}}$ by $\left\|\mathbf{u}_{1}\right\|_{L^{p}(\Omega)}^{\frac{2 p}{p-3}}$, and eventually $\left\|\Delta \mathbf{d}_{1}\right\|_{L^{p}(\Omega)}^{\frac{2 q}{2 q 3}}$ by $\left\|\nabla \mathbf{d}_{1}\right\|_{L^{p}(\Omega)}^{\frac{2 p}{p-3}}$. Therefore, the uniqueness is hold if we impose the additional hypothesis $(H 2)$ for $\mathbf{u}_{1}$, and $(H 3)$ or $(H 4)$ for $\mathbf{d}_{1}$.

\section{A Auxiliary results}

\section{A.1 Proof of Lemma 2.1:}

Multiplying the $\mathbf{d}$-system by $\mathbf{d}$, we obtain:

$$
\frac{1}{2} \partial_{t}\left(|\mathbf{d}|^{2}\right)+\gamma|\nabla \mathbf{d}|^{2}-\frac{\gamma}{2} \Delta\left(|\mathbf{d}|^{2}\right)+\gamma \mathbf{f}_{\delta}(\mathbf{d}) \cdot \mathbf{d}+(\mathbf{u} \cdot \nabla)\left(\frac{|\mathbf{d}|^{2}}{2}\right)=0 \quad \text { in } Q .
$$


Therefore, it is easy to deduce that:

$$
\partial_{t}\left(|\mathbf{d}|^{2}-1\right)+2 \gamma \mathbf{f}_{\delta}(\mathbf{d}) \cdot \mathbf{d}-\gamma \Delta\left(|\mathbf{d}|^{2}-1\right)+(\mathbf{u} \cdot \nabla)\left(|\mathbf{d}|^{2}-1\right) \leq 0 \quad \text { in } Q .
$$

Now, taking the inner product in $L^{2}(\Omega)$ with $\left(|\mathbf{d}|^{2}-1\right)_{+}:=\psi(\mathbf{d})$, using that:

$$
\left(\mathbf{f}_{\delta}(\mathbf{d}) \cdot \mathbf{d}\right)\left(|\mathbf{d}|^{2}-1\right)_{+} \geq 0
$$

and that

$$
\int_{\Omega}(\mathbf{u} \cdot \nabla)\left(|\mathbf{d}|^{2}-1\right) \psi(\mathbf{d}) d \Omega=0
$$

we obtain the inequality:

$$
\frac{d}{d t}\|\psi(\mathbf{d})\|_{L^{2}(\Omega)}^{2}+\gamma\|\nabla \psi(\mathbf{d})\|_{L^{2}(\Omega)}^{2}-\gamma \int_{\partial \Omega} \nabla \psi(\mathbf{d}) \cdot \mathbf{n} \psi(\mathbf{d}) d \sigma \leq 0 \quad \text { in }(0, T) .
$$

The boundary integral is zero since $\left.\psi(\mathbf{d})\right|_{\partial \Omega}=0$ in the Dirichlet case, and $\left.\nabla \psi(\mathbf{d}) \cdot \mathbf{n}\right|_{\partial \Omega}=0$ in the Neumann case. Then,

$$
\frac{d}{d t}\|\psi(\mathbf{d})\|_{L^{2}(\Omega)}^{2}+\gamma\|\nabla \psi(\mathbf{d})\|_{L^{2}(\Omega)}^{2} \leq 0
$$

As $\psi\left(\mathbf{d}_{0}\right)=\left(\left|\mathbf{d}_{0}\right|^{2}-1\right)_{+}=0$ in $\Omega$, therefore $\psi(\mathbf{d}) \equiv 0$, and thus $\left(|\mathbf{d}|^{2}-1\right)_{+}=0$. Hence, $|\mathbf{d}| \leq 1$ in $Q$.

\section{A.2 Proof of Lemma 2.2:}

We focus on the inequality verified when taking $A \mathbf{u}$ as a test function in $(L C)_{1}$ (which corresponds with the Prodi's estimates):

$$
\frac{d}{d t}\|\nabla \mathbf{u}\|_{L^{2}(\Omega)}^{2}+\|A \mathbf{u}\|_{L^{2}(\Omega)}^{2} \leq\|(\mathbf{u} \cdot \nabla) \mathbf{u}\|_{L^{2}(\Omega)}^{2}+\left\|(\nabla \mathbf{d})^{t} \Delta \mathbf{d}\right\|_{L^{2}(\Omega)}^{2}
$$

Now, we bound the terms on the right hand side of (38) as follows:

- for $q=3$, we have:

$$
\|(\mathbf{u} \cdot \nabla) \mathbf{u}\|_{L^{2}(\Omega)}^{2} \leq C\|\mathbf{u}\|_{L^{6}(\Omega)}^{2}\|\nabla \mathbf{u}\|_{L^{3}(\Omega)}^{2} \leq C\|\nabla \mathbf{u}\|_{L^{3}(\Omega)}^{2}\|\nabla \mathbf{u}\|_{L^{2}(\Omega)}^{2}
$$


- for $2 \leq q<3$, using the same interpolation estimates than in (14), we have:

$$
\begin{aligned}
\|(\mathbf{u} \cdot \nabla) \mathbf{u}\|_{L^{2}(\Omega)}^{2} & =\int_{\Omega}|\mathbf{u}|^{2}|\nabla \mathbf{u}|^{2} d \Omega \leq C\|\nabla \mathbf{u}\|_{L^{q}(\Omega)}^{2}\|\mathbf{u}\|_{L^{\frac{2 q}{q^{-2}}(\Omega)}}^{2} \\
& \leq C\|\nabla \mathbf{u}\|_{L^{q}(\Omega)}^{2}\|\nabla \mathbf{u}\|_{L^{5 q-6}(\Omega)}^{2} \\
& \leq C\|\nabla \mathbf{u}\|_{L^{q}(\Omega)}^{2}\|\nabla \mathbf{u}\|_{L^{2}(\Omega)}^{2 \alpha}\|\Delta \mathbf{u}\|_{L^{2}(\Omega)}^{2(1-\alpha)} \\
& \leq \bar{\varepsilon}\|\Delta \mathbf{u}\|_{L^{2}(\Omega)}^{2}+C_{\bar{\varepsilon}}\|\nabla \mathbf{u}\|_{L^{q}(\Omega)}^{2 / \alpha}\|\nabla \mathbf{u}\|_{L^{2}(\Omega)}^{2}
\end{aligned}
$$

where $\frac{2}{\alpha}=\frac{2 q}{2 q-3}$

On the other hand,

$$
\left\|(\nabla \mathbf{d})^{t} \Delta \mathbf{d}\right\|_{L^{2}(\Omega)}^{2} \leq C\|\nabla \mathbf{d}\|_{L^{6}(\Omega)}^{2}\|\Delta \mathbf{d}\|_{L^{3}(\Omega)}^{2} \leq C\|\nabla \mathbf{d}\|_{H^{1}(\Omega)}^{3}\|\Delta \mathbf{d}\|_{H^{1}(\Omega)} \in L^{2}(0, T)
$$

applying the regularity $\mathbf{d} \in L^{\infty}\left(0, T ; \mathbf{H}^{2}(\Omega)\right) \cap L^{2}\left(0, T ; \mathbf{H}^{3}(\Omega)\right)$.

Hence, using the Gronwall's Lemma we have that:

$$
\mathbf{u} \in L^{\infty}\left(0, T ; \mathbf{H}^{1}(\Omega)\right) \cap L^{2}\left(0, T ; \mathbf{H}^{2}(\Omega)\right)
$$

\section{A.3 Proof of Lemma 2.3:}

In order to estimate $\partial_{t} \mathbf{d}$, we use the equation verified by $\mathbf{d}$ :

$$
\partial_{t} \mathbf{d}-\gamma \Delta \mathbf{d}+(\mathbf{u} \cdot \nabla) \mathbf{d}=-\gamma \mathbf{f}_{\delta}(\mathbf{d})
$$

From the assumed regularity, we know that $\Delta \mathbf{d} \in L^{\infty}\left(0, T ; \mathbf{L}^{2}(\Omega)\right) \cap L^{2}\left(0, T ; \mathbf{H}^{1}(\Omega)\right)$. Next, we prove that the term $(\mathbf{u} \cdot \nabla) \mathbf{d}$ belongs to the same space:

$$
\begin{gathered}
\int_{\Omega}|\mathbf{u}|^{2}|\nabla \mathbf{d}|^{2} d \Omega \leq\|\mathbf{u}\|_{L^{3}(\Omega)}^{2}\|\nabla \mathbf{d}\|_{L^{6}(\Omega)}^{2} \leq\|\mathbf{u}\|_{L^{2}(\Omega)}\|\nabla \mathbf{u}\|_{L^{2}(\Omega)}\|\Delta \mathbf{d}\|_{L^{2}(\Omega)}^{2} \in L^{\infty}(0, T), \\
\int_{\Omega}|\mathbf{u}|^{2}\left|D^{2} \mathbf{d}\right|^{2} d \Omega \leq\|\mathbf{u}\|_{L^{6}(\Omega)}^{2}\|\Delta \mathbf{d}\|_{L^{3}(\Omega)}^{2} \leq\|\nabla \mathbf{u}\|_{L^{2}(\Omega)}^{2}\|\mathbf{d}\|_{H^{2}(\Omega)}\|\mathbf{d}\|_{H^{3}(\Omega)} \in L^{2}(0, T) \\
\int_{\Omega}|\nabla \mathbf{u}|^{2}|\nabla \mathbf{d}|^{2} d \Omega \leq\|\nabla \mathbf{u}\|_{L^{6}(\Omega)}^{2}\|\nabla \mathbf{d}\|_{L^{3}(\Omega)}^{2} \leq\|\Delta \mathbf{u}\|_{L^{2}(\Omega)}^{2}\|\nabla \mathbf{d}\|_{L^{2}(\Omega)}\|\mathbf{d}\|_{H^{2}(\Omega)} \in L^{1}(0, T)
\end{gathered}
$$


Finally, $\mathbf{f}_{\delta}(\mathbf{d}) \in L^{\infty}\left(0, T ; \mathbf{H}^{2}(\Omega)\right) \cap L^{2}\left(0, T ; \mathbf{H}^{3}(\Omega)\right)$, because of:

$$
\left\{\begin{array}{c}
\left\|\mathbf{f}_{\delta}(\mathbf{d})\right\|_{L^{2}(\Omega)}^{2} \leq C\|\mathbf{d}\|_{L^{2}(\Omega)}^{2} \\
\left\|\nabla \mathbf{f}_{\delta}(\mathbf{d})\right\|_{L^{2}(\Omega)}^{2} \leq C\|\nabla \mathbf{d}\|_{L^{2}(\Omega)}^{2} \\
\left\|D^{2} \mathbf{f}_{\delta}(\mathbf{d})\right\|_{L^{2}(\Omega)}^{2} \leq C\left\|D^{2} \mathbf{d}\right\|_{L^{2}(\Omega)}^{2}
\end{array}\right.
$$

Therefore, we can conclude that $\partial_{t} \mathbf{d} \in L^{\infty}\left(0, T ; \mathbf{L}^{2}(\Omega)\right) \cap L^{2}\left(0, T ; \mathbf{H}^{1}(\Omega)\right)$.

Now, we focus our attention on $\partial_{t} \mathbf{u}$. Taking $\partial_{t} \mathbf{u}$ as a test function in the system for $\mathbf{u}$, we obtain:

$$
\left\|\partial_{t} \mathbf{u}\right\|_{L^{2}(\Omega)}^{2}+\nu \frac{d}{d t}\|\nabla \mathbf{u}\|_{L^{2}(\Omega)}^{2} \leq C\left[\int_{\Omega}|\mathbf{u}|^{2}|\nabla \mathbf{u}|^{2} d \Omega+\int_{\Omega}|\nabla \mathbf{d}|^{2}|\Delta \mathbf{d}|^{2} d \Omega\right]
$$

We bound the terms on the right hand side as follows:

$$
\begin{aligned}
\int_{\Omega}|\mathbf{u}|^{2}|\nabla \mathbf{u}|^{2} d \Omega & \leq\|\mathbf{u}\|_{L^{3}(\Omega)}^{2}\|\nabla \mathbf{u}\|_{L^{6}(\Omega)}^{2} \\
& \leq\|\mathbf{u}\|_{L^{2}(\Omega)}\|\nabla \mathbf{u}\|_{L^{2}(\Omega)}\|\Delta \mathbf{u}\|_{L^{2}(\Omega)}^{2} \in L^{1}(0, T) \\
\int_{\Omega}|\nabla \mathbf{d}|^{2}|\Delta \mathbf{d}|^{2} d \Omega & \leq\|\nabla \mathbf{d}\|_{L^{3}(\Omega)}^{2}\|\Delta \mathbf{d}\|_{L^{6}(\Omega)}^{2} \\
& \leq\|\nabla \mathbf{d}\|_{L^{2}(\Omega)}\|\Delta \mathbf{d}\|_{L^{2}(\Omega)}\|\Delta \mathbf{d}\|_{H^{1}(\Omega)}^{2} \in L^{1}(0, T)
\end{aligned}
$$

Then, applying the Gronwall's Lemma, we conclude that $\partial_{t} \mathbf{u} \in L^{2}\left(0, T ; \mathbf{L}^{2}(\Omega)\right)$. Finally, we look again at the equation for $\mathbf{u}$ and we can easily deduce that $\nabla p \in L^{2}\left(0, T ; \mathbf{L}^{2}(\Omega)\right)$, and, therefore, that $p \in L^{2}\left(0, T ; H^{1}(\Omega)\right)$.

\section{A.4 Proof of Lemma 3.1:}

Thanks to the Lemmas 2.1 and 2.2 , it suffices to prove that $\mathbf{u} \in L^{2}\left(0, T ; \mathbf{H}^{2}(\Omega)\right)$ and $\mathbf{d} \in L^{2}\left(0, T ; \mathbf{H}^{3}(\Omega)\right)$. To this aim, we argue as follows:

1. We obtain the regularity $\Delta \mathbf{d} \in L^{2}\left(0, T ; \mathbf{H}^{1}(\Omega)\right)$ using the Prodi's estimates for $\mathbf{d}$.

2. Once the regularity for $\mathbf{d}$ is known, we obtain $\mathbf{u} \in L^{2}\left(0, T ; \mathbf{H}^{2}(\Omega)\right)$, using also the Prodi's estimates for $\mathbf{u}$. 
Estimates for $\Delta \mathbf{d}$ : In the homogeneous Neumann boundary condition case, we take $\Delta^{2} \mathbf{d}$ as test functions in the $\mathbf{d}$-system, obtaining:

$$
\begin{aligned}
\frac{1}{2} \frac{d}{d t}\|\Delta \mathbf{d}\|_{L^{2}(\Omega)}^{2} & +\gamma\|\nabla(\Delta \mathbf{d})\|_{L^{2}(\Omega)}^{2} \leq \int_{\Omega}|\nabla \mathbf{u}\|\nabla \mathbf{d}\| \nabla(\Delta \mathbf{d})| d \Omega \\
& +\int_{\Omega}|\nabla \mathbf{u} \| \Delta \mathbf{d}|^{2} d \Omega+\gamma \int_{\Omega} \nabla\left(\mathbf{f}_{\delta}(\mathbf{d})\right) \cdot \nabla(\Delta \mathbf{d}) d \Omega
\end{aligned}
$$

For the first term we have (using hypothesis $\|\nabla \mathbf{u}\|_{L^{2}(\Omega)} \leq C$ and $\left.\|\nabla \mathbf{d}\|_{H^{1}(\Omega)} \leq C\right)$ :

$$
\begin{aligned}
\int_{\Omega}|\nabla \mathbf{u}\|\nabla \mathbf{d}\| \nabla(\Delta \mathbf{d})| d \Omega & \leq C\|\nabla \mathbf{u}\|_{L^{2}(\Omega)}\|\nabla \mathbf{d}\|_{L^{\infty}(\Omega)}\|\nabla(\Delta \mathbf{d})\|_{L^{2}(\Omega)} \\
& \leq C\|\nabla \mathbf{d}\|_{H^{1}(\Omega)}^{1 / 2}\|\nabla \mathbf{d}\|_{H^{2}(\Omega)}^{1 / 2}\|\nabla(\Delta \mathbf{d})\|_{L^{2}(\Omega)} \\
& \leq C\|\nabla \mathbf{d}\|_{H^{2}(\Omega)}^{3 / 2} \leq \bar{\varepsilon}\|\nabla \mathbf{d}\|_{H^{2}(\Omega)}^{2}+C_{\bar{\varepsilon}} .
\end{aligned}
$$

In a similar way, we obtain for the second term:

$$
\begin{aligned}
\int_{\Omega}|\nabla \mathbf{u} \| \Delta \mathbf{d}|^{2} d \Omega & \leq\|\nabla \mathbf{u}\|_{L^{2}(\Omega)}\|\Delta \mathbf{d}\|_{L^{4}(\Omega)}^{2} \leq C\|\Delta \mathbf{d}\|_{L^{2}(\Omega)}^{1 / 2}\|\Delta \mathbf{d}\|_{H^{1}(\Omega)}^{3 / 2} \\
& \leq C\|\Delta \mathbf{d}\|_{H^{1}(\Omega)}^{3 / 2} \leq \bar{\varepsilon}\|\Delta \mathbf{d}\|_{H^{1}(\Omega)}^{2}+C_{\bar{\varepsilon}}
\end{aligned}
$$

And the last term can be bound as follows:

$$
\gamma \int_{\Omega} \nabla\left(\mathbf{f}_{\delta}(\mathbf{d})\right) \cdot \nabla(\Delta \mathbf{d}) d \Omega \leq \bar{\varepsilon}\|\nabla(\Delta \mathbf{d})\|_{L^{2}(\Omega)}^{2}+C_{\bar{\varepsilon}}\|\nabla \mathbf{d}\|_{L^{2}(\Omega)}^{2}
$$

Therefore, integrating in time (39), we arrive at $\mathbf{d} \in L^{2}\left(0, T ; \mathbf{H}^{3}(\Omega)\right)$.

The estimates for the nonhomogeneous Dirichlet boundary condition case do not present any additional difficulty.

Estimates for u: In the case of Prodi's estimates for $\mathbf{u}$, we take $A \mathbf{u}$ as test functions, obtaining :

$$
\frac{d}{d t}\|\nabla \mathbf{u}\|_{L^{2}(\Omega)}^{2}+\|A \mathbf{u}\|_{L^{2}(\Omega)}^{2} \leq \int_{\Omega}|\mathbf{u}|^{2}|\nabla \mathbf{u}|^{2} d \Omega+\int_{\Omega}|\nabla \mathbf{d}|^{2}|\Delta \mathbf{d}| d \Omega
$$

The terms on the right hand side can be bound in the following way:

$$
\begin{aligned}
\int_{\Omega}|\mathbf{u}|^{2}|\nabla \mathbf{u}|^{2} d \Omega & \leq\|\mathbf{u}\|_{L^{\infty}(\Omega)}^{2}\|\nabla \mathbf{u}\|_{L^{2}(\Omega)}^{2} \leq C\|\mathbf{u}\|_{H^{1}(\Omega)}\|\mathbf{u}\|_{H^{2}(\Omega)} \\
& \leq C\|A \mathbf{u}\|_{L^{2}(\Omega)} \leq \bar{\varepsilon}\|A \mathbf{u}\|_{L^{2}(\Omega)}^{2}+C_{\bar{\varepsilon}}
\end{aligned}
$$




$$
\begin{aligned}
\int_{\Omega}|\nabla \mathbf{d}|^{2}|\Delta \mathbf{d}|^{2} d \Omega & \leq\|\nabla \mathbf{d}\|_{L^{\infty}(\Omega)}^{2}\|\Delta \mathbf{d}\|_{L^{2}(\Omega)}^{2} \leq C\|\nabla \mathbf{d}\|_{H^{1}(\Omega)}\|\nabla \mathbf{d}\|_{H^{2}(\Omega)} \\
& \leq C\|\nabla \mathbf{d}\|_{H^{2}(\Omega)} \leq C\|\mathbf{d}\|_{H^{3}(\Omega)}
\end{aligned}
$$

Using the regularity obtained previously, we can conclude that $\mathbf{u} \in L^{2}\left(0, T ; \mathbf{H}^{2}(\Omega)\right)$.

\section{Acknowledgements}

The authors have been partially supported by the project BFM2003-06446-C02-01, and the third author by the project CNPq-Brasil 3013541-03-0.

\section{References}

[1] H. Beirã da Veiga, A new regularity class for the Navier-Stokes equations in $\mathbb{R}^{n}$. Chinese Ann. Math. 16 (1995), no. 4, 407-412.

[2] H. Beirã da Veiga, A sufficient condition on the pressure for the regularity of weak solutions to the Navier-Stokes equations. J. Math. Fluid Mech. 2 (2000), 99-106.

[3] L. C. Berselli, On a Regularity Criterion for the Solutions to the 3D Navier-Stokes Equations, Diff. and Integral Eq., Vol. 15, Number 9, 1129-1137 (2002).

[4] L. C. Berselli \& G. P. Galdi, Regularity criteria involving the pressure for the weak solutions to the Navier-Stokes equations, Proc. Am. Math. Society, Vol. 130, No. 12, 3585-3595 (2002).

[5] B. Climent, F . Guillén-González \& M. A. Rojas-Medar, Reproductivity solutions for a nematic liquid crystal model. Submitted.

[6] A. Friedman, Partial Differential Equations, Malabar Florida, 1983.

[7] Y. Giga, Solutions for semilinear parabolic equations in $L^{p}$ and regularity of weak solutions of the Navier-Stokes system. J. Differential Equations 62 (1986), no. 2, 186-212. 
[8] V. Girault \& F. Guillén-González, Mixed formulation, approximation and decoupling algorithm for a nematic liquid crystal model. In preparation.

[9] F. Guillén-González \& M. A. Rojas-Medar, Global solution of nematic liquid crystals models. C. R. Acad. Sci. Paris, Ser. I 335, 1085-1090 (2002).

[10] C. He, Regularity for solutions to the Navier-Stokes equations with one velocity component regular. Electron J. Differential Equations 29 (2002), 1-13.

[11] F.H. Lin, Nonlinear theory of defects in nematic liquid crystals: phase transition and flow phenomena. Comm. Pure Appl. Math. 42, 789-814 (1989).

[12] F.H. Lin \& C. Liu, Nonparabolic Dissipative Systems Modeling the Flow of Liquid Crystals. Communications on Pure and Applied Mathematics, Vol. XLVIII, 501-537 (1995).

[13] J.L. Lions, Quelques Méthodes de Résolution des Problèmes aux Limites Non Linéaires, Dunod, Paris, 1969.

[14] J.L. Lions \& E. Magenes, Problèmes aux limites non homogènes et applications, Vol. 1, Dunod, Paris, 1968.

[15] C. Liu \& J. Shen, On liquid crystal flows with free-slip boundary conditions. Discrete and Continuous Dynamical Systems, Vol. 7, No. 2, 2001.

[16] P. L. Lions, Mathematical Topics in Fluid Dynamics, Vol. 2 Compressible Models, Oxford University Press (1998).

[17] P. Penel \& M. Pokorný, Some new regularity criteria for the Navier-Stokes equations containing gradient of the velocity. Applications of Mathematics, 49 (2004), No. 5, 483-493.

[18] J. Serrin, On the interior regularity of weak solutions of the Navier-Stokes equations, Arch. Rat. Mech. Anal. 9 (3), 187-195 (1962). 